\title{
A new dust cycle model with dynamic vegetation: LPJ-dust version 1.0
}

\author{
S. Shannon and D. J. Lunt \\ Bristol Research Initiative for the Dynamic Global Environment (BRIDGE), School of Geographical Sciences, \\ University Road, University of Bristol, Bristol, BS8 1SS, UK
}

Received: 7 April 2010 - Published in Geosci. Model Dev. Discuss.: 23 April 2010

Revised: 20 December 2010 - Accepted: 20 January 2011 - Published: 16 February 2011

\begin{abstract}
This paper presents a new offline dust cycle model which uses the Lund-Potsdam-Jena dynamic global vegetation model (Sitch et al., 2003) to calculate time varying dust sources. Surface emissions are calculated by simulating the processes of saltation and sandblasting using an existing model (Tegen et al., 2002). Dust particles are transported using the TOMCAT chemical transport model (Chipperfield, 2006). Dust particles are removed from the atmosphere by dry deposition and sub-cloud scavenging. The model is designed so that it can be driven using reanalysis data or GCM derived fields.

To improve the performance of the model, threshold values for vegetation cover, soil moisture, snow depth and threshold friction velocity, used to determine surface emissions are tuned. The effectiveness of three sub-cloud scavenging schemes are also tested. An ensemble of tuning experiments are evaluated against dust deposition and surface concentration measurements. Surface emissions which produce the best agreement with observations range from 1600 to $2400 \mathrm{Mtyr}^{-1}$.
\end{abstract}

\section{Introduction}

Mineral dust plays an interactive role in the Earth's system by modifying the radiation balance (Forster et al., 2007) and transporting nutrients to the terrestrial (Kaufman et al., 2005; Menendez et al., 2007) and marine ecosystems (Coale et al., 2004; Jickells et al., 2005). Observations show that vegetation cover may play a role in constraining dust emissions on seasonal and inter-annual time scales (Zhao, 2004; Lee and Sohn, 2009). In the Sahel, a three way connection between

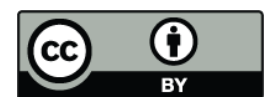

Correspondence to: S. Shannon (sarah.shannon@bristol.ac.uk) rainfall, vegetation and dust emissions has been suggested, whereby a decrease in precipitation in the Sahel causes a reduction in vegetation cover, which increases dust emissions (Evan et al., 2006; Zender and Kwon, 2005).

This theory is supported by measurements of Normalised Difference Vegetation Index (NDVI) in the Sahel which show that vegetation cover responds to changes in precipitation (Tucker et al., 1991). Studies have shown that this response occurs relatively quickly. NDVI has been correlated with rainfall for the concurrent month plus the two previous months (Nicholson et al., 1990; Herrmann et al., 2005). Limitations in vegetation models means that dust cycle models are unable to simulate this fast response.

Two categories of dust cycle models have been developed to date; models which use remote sensing data to describe vegetation cover on the land surface (e.g. Zender et al., 2003; Ginoux et al., 2004; Grini et al., 2005; Cakmur et al., 2006) and models which use vegetation models, typically Equilibrium Biogeography-Biogeochemistry models (BIOME3 or BIOME4) to simulate the distribution of vegetation cover (e.g. Werner et al., 2002; Mahowald et al., 2002; Lunt and Valdes, 2002; Mahowald et al., 1999). The latter category can be used as predictive tools to estimate how the dust loading will change in the future or in the past under different climatic conditions.

Dust cycle models which use BIOME3 or BIOME4 are unable to simulate the inter-annual variability in dust source areas caused by the dynamic response of vegetation cover to the climate. As a consequence, it is not possible to test whether changes in the dust loading are caused by variability in vegetation cover or by other processes. For this reason this work describes a dust cycle model which uses the LundPotsdam-Jena dynamic global vegetation model (LPJ) (Sitch et al., 2003) to simulate the dynamic vegetation on the land surface.

Published by Copernicus Publications on behalf of the European Geosciences Union. 
As with any numerical model of a physical system, uncertainty in the model results will arise from parametric and structural uncertainty and uncertainty in the input data used to drive the model. Parametric uncertainty in a dust model may be associated with the values for threshold limits for vegetation cover, soil moisture, snow cover and threshold friction velocity used to calculate surface emissions. Lunt and Valdes (2002) showed that the dust loading is very sensitive to the choice of values for these thresholds. For example, they found that increasing the threshold friction velocity from 0.4 to $0.6 \mathrm{~ms}^{-1}$ caused a decrease in the dust loading by a factor of 19 .

A way to constrain the threshold limits is to perform a model tuning. One strategy for tuning is to produce an ensemble of models by selecting certain values for model parameters and selecting from these a subset of models which perform well compared to observations. A way to select values for parameters is to use Latin Hypercube Sampling (McKay et al., 1979). This approach has been taken by Edwards and Marsh (2005) to tune parameters in a 3-D ocean climate model and by Schneider von Deimling et al. (2006) to tune parameters in the CLIMBER-2 intermediate complexity climate model. The technique divides each tunable parameter into equal intervals $(\mathrm{N})$ of equal probability $(1 / \mathrm{N})$. One sample is selected at random from each interval and matched up randomly with a sample selected for another parameter. The advantage of this technique over randomly choosing values is that it ensures that all regions of parameter space are evenly sampled. In this paper, Latin Hypercube Sampling is used to select values for tuneable parameters in the model.

A source of structural uncertainty in the model arises from the choice of parameterisation for sub-cloud scavenging. Jung and Shao (2006) examined the characteristics of four different sub-cloud scavenging schemes within the framework of a dust cycle model. They found that the choice of sub cloud scavenging scheme affected the ability of the model to accurately predict surface concentrations of dust at selected locations in Asia. Furthermore, the scavenging coefficient deviated by a factor of 1000 depending on the precipitation rate and particle size. To reduce the structural uncertainty associated with wet deposition three sub-cloud scavenging schemes are tested in this paper as part of the model tuning.

This paper presents a description of the new dust cycle model and tuning. The layout of the paper is as follows: in Sect. 2, the dust model is described. This includes details of how dust source areas are calculated from LPJ, a description of the dust emission scheme, the chemical transport model and parameterisation of wet and dry deposition. A baseline dust simulation is described in Sect. 2.4. The method used for selecting values for threshold parameters is described in Sect. 2.5. The three types of sub-cloud scavenging schemes are described in Sect. 2.6. The measurement datasets used to evaluate the model performance are described in Sect. 2.7. Finally, the results of the model tuning and potential applications of the model are discussed in Sect. 3.

\section{Dust model description}

The dust model comprises of three existing models. LPJ (Sitch et al., 2003) is used to calculate the distribution of unvegetated areas which may act as potential dust sources. This is linked to an existing model which calculates dust emissions by simulating the processes of saltation and sandblasting (Tegen et al., 2002). Dust particles are transported as independent tracers within the TOMCAT chemical transport model (Chipperfield, 2006). Dust is removed from the atmosphere by dry deposition and sub-cloud scavenging. The following section describes the components of the dust model (Fig. 1).

\subsection{Calculation of dust source areas using LPJ}

LPJ simulates vegetation dynamics by modeling the atmosphere-vegetation carbon and water fluxes, plant physiology, phenology, establishment and mortality. LPJ calculates daily gross primary production (GPP) by modeling the processes of photosynthesis and transpiration using a coupled photosynthesis and water balance scheme developed in the BIOME3 model (Haxeltine and Prentice, 1996). A fraction of the GPP produced is used for the plant respiration. The remaining fraction known as the net primary production (NPP) is allocated to the leaf, sapwood and fine root carbon pools, satisfying a series of structural constraints.

Vegetation is grouped into ten plant functional types (PFTs) which are categorised according to their plant physiological (C3, C4 photosynthesis), phenological (deciduous, evergreen) and physiognomic (tree, grass) attributes. Plant mortality by fire, heat stress, competition for light and whether there is insufficient carbon to grow is modeled on an annual basis. Every year a proportion of the total vegetation cover decomposes and falls to the surface as litter and new vegetation is established. A set of bioclimatic limits are used to determine if a PFT can survive within a particular temperature range. The establishment of new PFTs is prohibited when the annual precipitation is less than $100 \mathrm{~mm} \mathrm{yr}^{-1}$.

Studies have been carried out to validate LPJ vegetation cover (Sitch et al., 2003) and hydrology (Wagner et al., 2003; Gerten et al., 2004). LPJ has been shown to successfully reproduce inter-annual variability in vegetation cover in the Sahel from 1980 to 2002 (Seaquist et al., 2009). Latitudinal shifts in vegetation cover in this region may affect the location of the Sahara-Sahel boundary line and thus influence the quantity of dust emitted from North Africa.

LPJ is forced using annual mean atmospheric $\mathrm{CO}_{2}$ and monthly mean precipitation, fractional cloud cover and temperature. In this paper, these are obtained from the Climate Research Unit, University of East Anglia, UK (CRU 2.1), but they could equally be obtained from a GCM. Historical $\mathrm{CO}_{2}$ data from 1901 to 1995 is obtained from the Carbon Cycle Model Linkage project (McGuire et al., 2001; Cramer et al., 1999). Information on soil texture is taken from the 


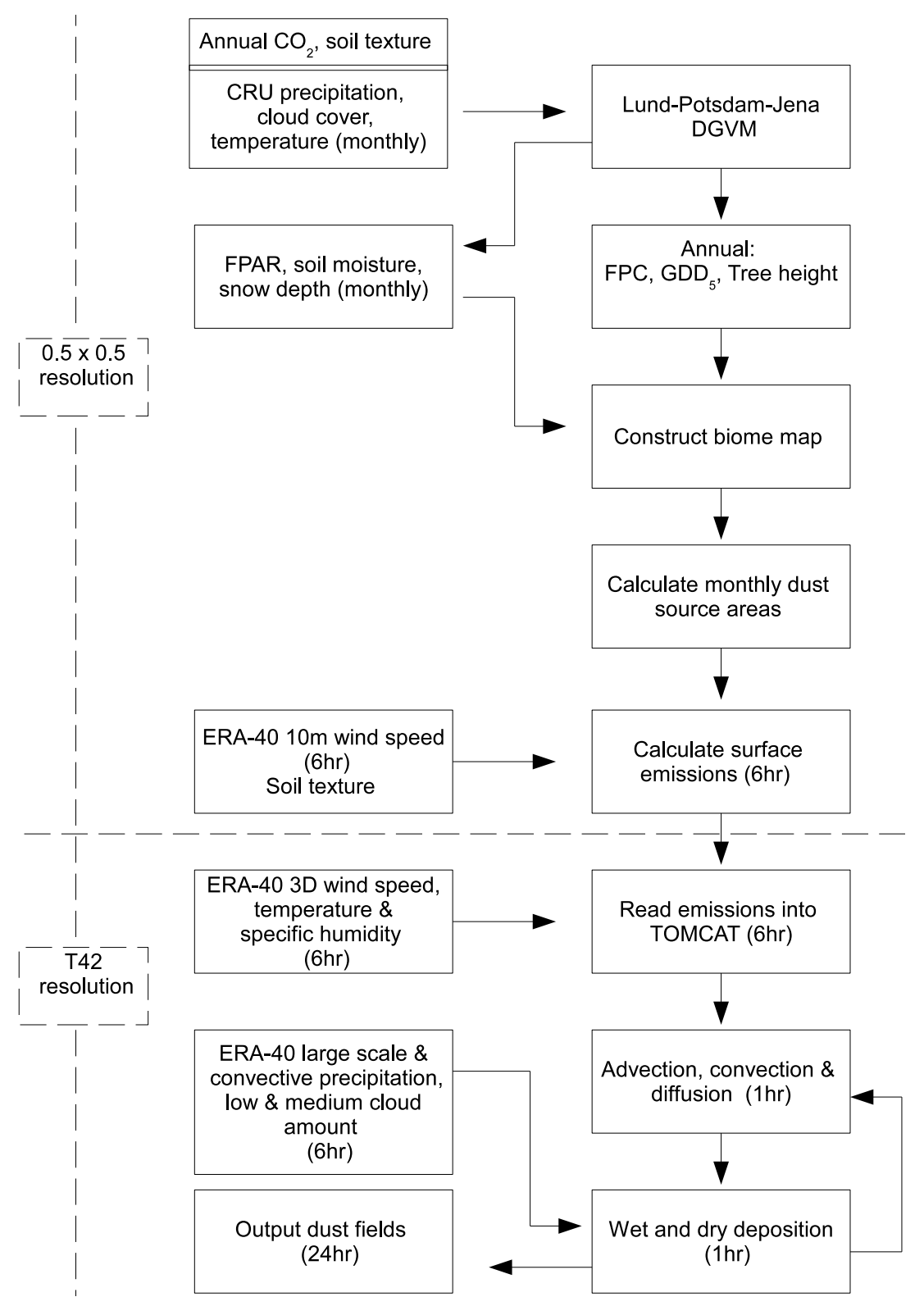

Fig. 1. A schematic of the LPJ-dust model and its components.

Soil Food and Agriculture Organization United Nations Educational, Scientific and Cultural Organization soil map of the world (Zobler, 1986). This is used to calculate the daily percolation of water from the upper soil layer to the lower soil layer.

LPJ is run on a $0.5 \times 0.5$ degree spatial resolution. The simulation begins with no vegetation cover and is allowed to spin up for 1000 years so that the vegetation cover and carbon pools reach equilibrium. This is achieved by forcing the model with the first 30 years of the CRU climate repetitively for 1000 years. The model is then forced using 102 years of the CRU climate data.
To demonstrate the relationship between vegetation cover, precipitation and dust source areas in the model, Fig. 1 shows the correlation between the annual mean fraction of photosynthetically active radiation (FPAR) simulated by LPJ and the CRU precipitation in the previous year. The correlation coefficient is calculated over the years 1958 to 2002 . Regions of high positive correlation are visible on the margins of dust sources. This signifies that vegetation cover responds to precipitation in these regions. Figure 2 shows the standard deviation of the un-vegetated area for the same time period, 


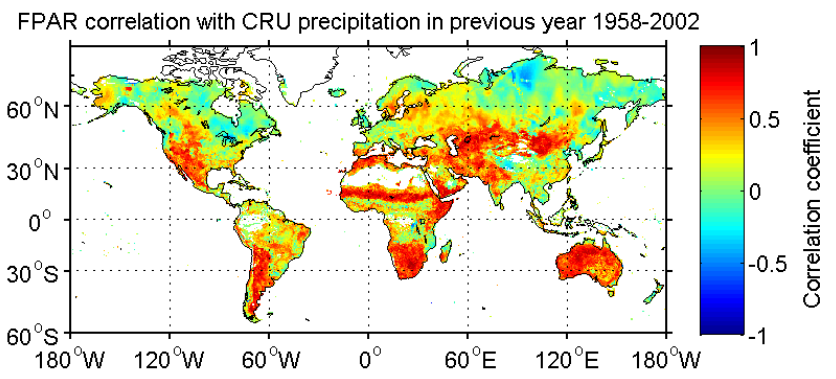

Fig. 2. Correlation between annual mean FPAR simulated by LPJ and $\mathrm{CRU}$ precipitation in the previous year.

where the un-vegetated area is

$$
A=1-\frac{\text { mfpar }}{\text { mfpar }_{\text {lim }}}
$$

and mfpar $_{l i m}$ is 0.5 and mfpar is the monthly FPAR. Interannual variability in the un-vegetated area is evident at the boundaries of the dust source regions.

\subsubsection{LPJ outputs used to calculate dust source areas}

The following variables are output annually and used to calculate monthly dust source areas:

1. Annual foliage projective cover (FPC).

The FPC is calculated from FPC $_{\mathrm{PFT}}$, where $\mathrm{FPC}_{\mathrm{PFT}}$ is the fractional coverage of each PFT in a grid cell. The FPC has a value of 1 if the grid cell is completely covered in vegetation or 0 if no vegetation cover is present. The FPC is calculated from the FPC $\mathrm{PFT}_{\mathrm{T}}$ using the following relationship.

$\sum_{\mathrm{PFT}=1}^{\mathrm{PFT}=10} \mathrm{FPC}=\mathrm{CA}(\mathrm{PFT}) \cdot P(\mathrm{PFT}) \cdot \mathrm{FPC}_{\mathrm{PFT}}$

where $\mathrm{CA}(\mathrm{PFT})$ is the crown area and $\mathrm{P}(\mathrm{PFT})$ is the population density of the PFT. The crown area is calculated using an empirical relationship between crown area and stem diameter (Zeide, 1993). The FPC $_{\mathrm{PFT}}$ is calculated using the following relationship (Monsi and Saeki, 1953).

$\mathrm{FPC}_{\mathrm{PFT}}=1-e^{-0.5 \mathrm{LAI}_{\mathrm{PFT}}}$

where LAIPFT is the leaf area index of the PFT which is related to the amount of carbon stored in the leaf.

2. Annual growing degree days base $5^{\circ} \mathrm{C}\left(\mathrm{GDD}_{5}\right)$.

$\mathrm{GDD}_{5}$ is calculated by summing the daily temperatures $T_{\mathrm{d}}$ when temperatures are greater than $5^{\circ} \mathrm{C}$

$\mathrm{GDD}_{5}=\left[\begin{array}{cc}\sum_{d=1}^{d=365} T_{\mathrm{d}} & \text { if } T_{\mathrm{d}}>5^{\circ} \mathrm{C} \\ 0 & \text { otherwise }\end{array}\right]$
$T_{\mathrm{d}}$ is calculated by interpolating monthly temperatures onto a daily time step.

3. Annual tree height $(H)$.

The annual tree height is calculated using the empirical relationship between vegetation height and stem diameter (Huang et al., 1992).

$H=k_{\text {allom } 2} D^{\text {allom } 3}$

where $k_{\text {allom } 2}=40$ and allom $3=0.5$ are constants and $D$ is the stem diameter.

4. Monthly volumetric soil moisture (sm).

The soil moisture in LPJ is calculated using a semi-empirical approach which was developed in the BIOME3 model (Haxeltine et al., 1996). The soil is divided into two layers of $0.5 \mathrm{~m}$ each. The water held in each layer is calculated daily by taking into account the precipitation, snow melt, percolation, evapotranspiration and runoff. The percolation rate is dependent on the soil texture. When the soil layer is at field capacity the excess water is considered to be runoff. The soil water content of the upper layer on any given day is related to the amount of water into the soil layer plus the water out of the soil layer during the previous day.

$\mathrm{sm}=\left(\right.$ melt + precip - perc - runoff $\left.-\beta_{1} \mathrm{AET}\right)-\mathrm{AWC}_{1}$

where melt is the snowmelt, precip is the precipitation, perc is the percolation, runoff is the runoff and $\beta_{1}$ is the rate of transpired water from the upper layer to the lower layer. AET is the calculated evapotranspiration rate for each plant functional type. $\mathrm{AWC}_{1}$ is the available water holding capacity. The soil moisture in the upper $0.5 \mathrm{~m}$ of the soil is converted from units of mm into percentage volumetric soil moisture.

5. Monthly snow depth (sd).

LPJ calculates monthly snow depth using daily precipitation data which is derived from monthly precipitation that has been interpolated onto a daily time step. When the daily temperature is less than $-2{ }^{\circ} \mathrm{C}$, new snow is formed. The magnitude of the snow formed is proportional to the daily precipitation. An adjustment is made to the snow depth to account for the melting of snow. Snow melt occurs when the daily temperature is greater than $-2{ }^{\circ} \mathrm{C}$. The amount of melting is related to the temperature by snow melt coefficient taken from the BIOME3 model (Haxeltine et al., 1996).

6. Monthly fraction of photosynthetically active radiation (mfpar).

The mfpar predicted by LPJ gives an indication of the state and productivity of the vegetation cover. This 
quantity is defined as the fraction of incoming solar radiation absorbed by vegetation cover which is used to drive photosynthesis. It is calculated using the following relationship,

$\operatorname{mfpar}=\mathrm{FPC}_{\mathrm{PFT}} \cdot D_{\mathrm{phen}}$

where $D_{\text {phen }}$ is the daily leaf-on fraction. $D_{\text {phen }}$ and is calculated from the accumulated $\mathrm{GDD}_{5}$ and has a value of 1 when leaves are present and 0 when leaves are absent.

\subsubsection{Creating a biome map of vegetation cover}

$\mathrm{GDD}_{5}$ and $H$ are used to convert FPC into a biome map every year using a scheme adapted from Joos et al. (2004). This conversion is carried out because at high latitudes, LPJ predicts barren land (i.e. $\mathrm{FPC}=0$ ), combined with low soil moisture and low snow cover which is a criteria for a dust source. This results in a large dust source area in the Canadian Arctic. Creating a biome map allows polar desert, which has low $\mathrm{GDD}_{5}$ and is not a dust source, to be distinguished from a hot desert which has high $\mathrm{GDD}_{5}$ and is a dust source. Using this scheme also allows trees with a stand height of less than $4 \mathrm{~m}$ to be considered as shrubs. Although this is a simplification, it means that regions with woody PFTs will act as dust sources if productivity is sufficiently low. This is a useful assumption as LPJ does not simulate shrub PFTs. A schematic of the scheme used to create a biome map is shown in Fig. 4. Dust emissions are permitted for regions containing hot desert, dry grass, dry shrubs, tundra grass and tundra shrubs.

\subsubsection{Calculating monthly dust source areas}

For grass-dominated biomes (tundra grass and dry grass) the area exposed for dust emission is allowed to vary seasonally. The un-vegetated area $A_{\text {grass }}$ is linearly proportional to the mfpar below a threshold value mfparlim.

$A_{\text {grass }}= \begin{cases}1-\frac{\mathrm{mfpar}}{\mathrm{mfpar}} & \text { if } \text { mfpar }<\text { mfpar }_{\text {lim }} \\ 0 & \text { otherwise }\end{cases}$

where mfpar is calculated from Eq. (6).

In shrub dominated biomes the area exposed for dust emission remains fixed throughout the year. This is because shrubs are assumed to protect the surface all year round even when no leaves are present. The annual maximum mfpar $\left(\operatorname{mfpar}_{\max }\right)$ is used as an index for the density of shrubs. For shrub dominated biomes, the area is calculated as

$A_{\text {shrub }}= \begin{cases}1-\text { mfpar }_{\text {max }} & \text { if mfpar }<\text { mfpar }_{\text {lim }} \\ 0 & \text { otherwise },\end{cases}$

This means the dust source area remains constant throughout the year but decreases to zero when the $\left(\operatorname{mfpar}_{\max }\right)=1$.
The same mfpar ${ }_{\text {lim }}$ is used for grasses and shrubs in the model. LPJ does not explicitly simulate shrub PFTs. The scheme used to convert PFTs into biomes produces very little dry shrub land compared to the Matthews vegetation map (Matthews, 1983). This is caused by the lack of PFTs with $\mathrm{GDD}_{5}>500$ and tree height $<4 \mathrm{~m}$ simulated by LPJ. The scheme is reasonably successful at predicting tundra shrub land. We would expect the mfpar ${ }_{\text {lim }}$ for dry shrubs to be higher than that used for grasses and tundra shrubs. However, because very little dry shrubs are simulated, we decided that using the same mfpar ${ }_{\text {lim }}$ would not significantly affect estimates of dust source regions.

At high latitudes, dust emissions are suppressed by snow cover. The area exposed for dust emission, $\mathrm{A}_{\text {snow }}$, is linearly related to the snow depth (sd) below a threshold value ( $\left.\mathrm{sd}_{\text {lim }}\right)$.

$A_{\text {snow }}= \begin{cases}1-\frac{\mathrm{sd}}{\mathrm{sd}_{\text {lim }}} & \text { if } \mathrm{sd}<\mathrm{sd}_{\text {lim }} \\ 0 & \text { otherwise }\end{cases}$

The total area available for dust emission is related to area of dry ground that is un-vegetated and not covered by snow. The erodible area $A_{\text {bare }}$ is expressed by the following form

$A_{\text {bare }}= \begin{cases}A_{\text {grass }} \cdot A_{\text {snow }} \cdot I_{\theta} & \text { for grass biomes } \\ A_{\text {shrub }} \cdot A_{\text {snow }} \cdot I_{\theta} & \text { for shrub biomes }\end{cases}$

where $A_{\text {grass }}$ and $A_{\text {shrub }}$ is the contribution of exposed ground from shrub or grass vegetation cover, $A_{\text {snow }}$ is the contribution from snow cover. $I_{\theta}$ represents the effect of the soil moisture. When sm exceeds a threshold limit $\mathrm{sm}_{\mathrm{lim}}$, then $I_{\theta}$ is assigned a value of 0 and no dust emissions occur. Conversely, if the soil moisture is below $\mathrm{sm}_{\mathrm{lim}}$, then $I_{\theta}$ has a value of 1 and dust emissions will occur.

\subsection{Calculation of the dust flux}

The calculation of the dust flux is taken from the model by Tegen et al. (2002). The model parameterises saltation and sandblasting using the scheme by Marticorena and Bergametti (1995). The horizontal flux $G_{j}$ generated by saltating particles is calculated as

$G_{j}=\frac{\rho_{\mathrm{a}}}{g} u^{* 3}\left(1+\frac{\eta u_{t}^{*}}{u^{*}}\right)\left(1-\frac{\left(\eta u_{t}^{*}\right)^{2}}{u^{* 2}}\right) \cdot s_{j}$

where $\rho_{\mathrm{a}}$ is the density of air $\left(\mathrm{kgm}^{-3}\right), g$ is the gravitational constant $\left(\mathrm{ms}^{-1}\right), u^{*}$ is the surface wind velocity $\left(\mathrm{ms}^{-1}\right)$ and $u_{t}^{*}$ is the threshold friction velocity $\left(\mathrm{ms}^{-1}\right)$.

$s_{j}$ is used to scale the relative contribution of each size fraction $j$ to the total flux. $s_{j}$ is the surface area covered by a particle size fraction relative to the area covered by the total flux of particles. The surface covered by each grain is calculated from its basal surface. This is related to the mass $(M)$ of the particle such that,

$d S\left(D_{\mathrm{p}}\right)=\frac{\mathrm{d} M\left(D_{\mathrm{p}}\right)}{\frac{2}{3} \rho_{\mathrm{p}} D_{\mathrm{p}}}$ 
where $\rho_{\mathrm{d}}$ is the density of the particle and $D_{\mathrm{p}}$ is the particle diameter. The total basal surface is

$S_{\text {TOTAL }}=\int_{D_{\mathrm{p}}} \mathrm{d} S\left(D_{\mathrm{p}}\right) \mathrm{d} D_{\mathrm{p}}$

The relative area covered by each particle fraction is then,

$s_{j}=\frac{d S\left(D_{\mathrm{p}}\right)}{S_{\mathrm{TOTAL}}}$

$u_{t}^{*}$ in Eq. (11) is calculated as a function of particle diameter using a semi-empirical relationship described by Iversen and White (1982). $u_{t}^{*}$ is modified to account for the presence of non-erodible elements such as vegetation cover or rocks on the surface. The adjustment to $u_{t}^{*}$ is applied by dividing by the drag partition ratio $f_{\text {eff }}$ (Marticorena and Bergametti, 1995)

$f_{\text {eff }}=1-\left[\frac{\ln \left(\frac{z_{0}}{z_{0 s}}\right)}{\ln \left(0.35\left(\frac{10}{z_{0 s}}\right)^{0.8}\right)}\right]$

where roughness length of a surface with no obstacles $z_{0 s}=$ $0.001 \mathrm{~cm}$. The roughness length of the surface $z_{0}$ is assigned a value of $0.01 \mathrm{~cm}$ which is a typical value for level desert (Seinfeld and Pandis, 1998). In this case $f_{\text {eff }}$ has a constant value of 0.64 for all dust emitting regions.

The friction velocity $u^{*}$ in Eq. (11) is calculated as a function of surface roughness, such that

$u^{*}=\frac{u}{k} \ln \left(\frac{z}{z_{0}}\right)$

where $k$ is the Von Karman constant $=0.4$ (dimensionless), $z$ is the height (m), $z_{0}$ is the roughness length (m) and $u\left(\mathrm{~ms}^{-1}\right)$ is the wind speed. $\eta$ in Eq. (11) is a tunable parameter which serves to increase or decrease $u_{t}^{*}$. The default value for $\eta$ used by Tegen et al. (2002) is 0.66 .

Dust emissions are calculated on a six hourly time step using ERA-40 $10 \mathrm{~m}$ wind speeds. The emissions are calculated on 0.5 degree $\times 0.5$ degree resolution to match the LPJ resolution. $1 \times 1$ degree wind speed data is interpolated onto the $0.5 \times 0.5$ degree resolution by assuming that four adjoining half degree pixels have the same wind speed as a 1 degree pixel.

The vertical flux $F$ is estimated from the horizontal flux by the following

$F=\alpha A_{\text {bare }} G$

where $G$ is the horizontal flux determined from Eq. (11), $A_{\text {bare }}$ is the monthly bare ground fraction which has been calculated from LPJ in Eq. (10) and $\alpha$ is the sandblasting mass efficiency. The $\alpha$ values used in the model are taken from Marticorena et al. (1997) who summarise the experimental values for different soil types. For completeness the $\alpha$ for different soil types are listed in Table 1.
Table 1. Column 2 contains the sandblasting mass efficiency values for different soil textures. Columns 3 to 6 contain the relative mass of the main soil types for each soil texture. These values are used to calculate the particle size distribution in Eq. (18).

\begin{tabular}{llllll}
\hline $\begin{array}{l}\text { Zobler texture } \\
\text { classes }\end{array}$ & $\alpha \mathrm{cm}^{-1}$ & $\begin{array}{l}\text { Coarse } \\
\text { Sand }\end{array}$ & $\begin{array}{l}\text { Medium/ } \\
\text { Fine } \\
\text { Sand }\end{array}$ & Silt & Clay \\
\hline Coarse & $2.1 \times 10^{-6}$ & 0.43 & 0.4 & 0.17 & - \\
Medium & $4.0 \times 10^{-6}$ & - & 0.37 & 0.33 & 0.3 \\
Fine & $1.0 \times 10^{-7}$ & - & - & 0.33 & 0.67 \\
Coarse-Medium & $2.7 \times 10^{-6}$ & 0.1 & 0.5 & 0.20 & 0.20 \\
Coarse-Fine & $2.8 \times 10^{-6}$ & 0 & 0.5 & 0.12 & 0.38 \\
Medium-Fine & $1.0 \times 10^{-7}$ & 0 & 0.27 & 0.27 & 0.48 \\
Coarse-Medium-Fine & $2.5 \times 10^{-6}$ & 0.23 & 0.23 & 0.19 & 0.35 \\
\hline
\end{tabular}

Information on the particle size distribution comes from the Soil Food and Agriculture Organization United Nations Educational, Scientific and Cultural Organization soil map of the world (Zobler, 1986). The particle size distribution for each soil texture type is calculated using the following relationship from Tegen et al. (2002)

$$
\begin{aligned}
& \frac{d M\left(D_{\mathrm{p}}\right)}{d \ln D_{\mathrm{p}}} \\
& \quad=\sum_{j=1}^{n} \frac{M_{j}}{(2 \pi)^{\frac{1}{2}} \ln \sigma_{j}} \exp \left(\frac{\left(\ln D_{\mathrm{p}}-\ln \mathrm{MMD}_{j}\right)^{2}}{-2 \ln ^{2} \sigma_{j}}\right)
\end{aligned}
$$

$D_{\mathrm{p}}$ is the particle size, $M_{j}$ is the percentage mass of coarse sand, medium/fine sand, silt or clay, $\mathrm{MMD}_{j}$ is the mass median diameter and $\sigma$ has a value of 2. The values from $M_{j}$ for each soil type are listed in Table 1.

Dust emissions are calculated for particles with diameter $0.1 \mu \mathrm{m}, 0.3 \mu \mathrm{m}, 0.9 \mu \mathrm{m}, 2.6 \mu \mathrm{m}, 8 \mu \mathrm{m}, 24 \mu \mathrm{m}, 72 \mu \mathrm{m}$ and $220 \mu \mathrm{m}$. The emissions are re-gridded from a $0.5 \times 0.5 \mathrm{spa}-$ tial resolution onto a T42 spatial resolution for input into the TOMCAT chemical transport model.

\subsection{Transport and removal}

Dust particles are transported as independent tracers using the chemical transport model TOMCAT (Chipperfield, 2006). TOMCAT is driven by 3 -D wind speeds, specific humidity and temperature which can be derived from either meteorological re-analysis data or GCM output. TOMCAT simulates the transport of gaseous or aerosol species via advection, convection and vertical diffusion.

The advection scheme used in TOMCAT is the conservation of second order moments developed by Prather (1986). The Prather advection scheme represents tracer concentration as second-order polynomials within each grid box. This makes the scheme more computationally expensive than simpler schemes, such as the slopes scheme by Russell and 
Lerner (1981). Although the Prather advection scheme is expensive, it has been shown to have low numerical diffusion, thus providing more accurate results (Ge and Lei, 1998).

Convection is parameterised in TOMCAT using a scheme by developed by Tiedtke (1989). The scheme includes cumulus updrafts in the vertical direction and the exchange of air from inside the cloud to outside the cloud and vice versa. The convective scheme calculates the mass of tracer that is uplifted within a cloud column. Vertical diffusion is parameterised in TOMCAT using a scheme developed by Louis (1979).

TOMCAT is forced using ERA-40 6 hourly 3-D temperature, $U$ and $V$ wind speed and specific humidity fields on a T42 spatial resolution. The model has 31 vertical pressure levels extending from the surface to the stratosphere. Advection, convection, diffusion and dust removal take place on an hourly time step.

\subsubsection{Dry deposition}

The dry deposition parameterisation consists of gravitational settling and turbulent mixing across the quasi sub-laminar layer. The dry deposition parameterisation is taken from Lunt (2001) which is based on equations for dry deposition described in Seinfeld and Pandis (1998). The rate of dust removal by dry deposition per unit area per unit time $F_{\mathrm{Z}}$ is proportional to the concentration of dust at a particular height $C_{\mathrm{Z}}$ and to the deposition velocity $v_{\mathrm{d}}$ by the following relationship,

$F_{\mathrm{z}}=v_{\mathrm{d}} C_{\mathrm{z}}$

The dry deposition process is conceptualised in terms of an electric circuit containing resistance in series. $r_{\mathrm{a}}$ is the aerodynamic resistance and $r_{\mathrm{b}}$ is the quasi laminar sub layer resistance. The total $v_{\mathrm{d}}$ is then

$v_{\mathrm{d}}=v_{\mathrm{s}}+\frac{1}{r_{\mathrm{a}}+r_{\mathrm{b}}+r_{\mathrm{a}} r_{\mathrm{b}} v_{\mathrm{s}}}$

The first term on the right hand side of the equation corresponds to the gravitational settling velocity $\left(v_{\mathrm{s}}\right)$. The second expression corresponds to the deposition velocity across the quasi laminar sub-layer.

The gravitational settling velocity $v_{\mathrm{s}}$ is

$v_{\mathrm{s}}=\frac{\rho_{\mathrm{p}} D_{\mathrm{p}}^{2} g C_{\mathrm{c}}}{18 \mu}$

where $\rho_{\mathrm{p}}$ is the density of the particle $\left(\mathrm{kgm}^{-3}\right), D_{\mathrm{p}}$ is the particle diameter $(\mathrm{m}), g$ is gravitational constant $\left(\mathrm{ms}^{-2}\right), \mu$ is the viscosity of air $\left(\mathrm{kg} \mathrm{ms}^{-1}\right)$ and $C_{\mathrm{c}}$ is the slip correction factor. $C_{\mathrm{c}}$ becomes important when the particle diameter approaches the same magnitude as the mean free path of air and the medium can no longer be considered a continuum. The slip correction factor is given by

$C_{\mathrm{c}}=1+\frac{2 \lambda}{D_{\mathrm{p}}}\left(1.257+0.4 \exp ^{-0.55 D_{\mathrm{p}} / \lambda}\right)$

where $\lambda$ is the mean free path of the air (m).

Dust is transported downwards by gravitational settling through each model vertical level. For simplification, we assume that particles do not fall though more than one vertical level within one time step.

For the shortest model level and a time step of one hour, the assumption implies that the deposition velocity should not exceed $18000 \mathrm{~cm} \mathrm{~h}^{-1}$. This applies to the six smallest particles sizes which have deposition velocities less than $16000 \mathrm{~cm} \mathrm{~h}^{-1}$. The two largest particle sizes fall faster and do not obey the assumption. These particles represent only $3.2 \%$ of the total surface emissions, therefore, we assume the simplification does not cause a large uncertainty in the model estimates of surface concentrations and deposition rates.

At the lowest model level the resistance of the quasi laminar sub-layer in Eq. (20) is defined as

$r_{\mathrm{b}}=\frac{1}{u^{*}\left(S c^{\frac{-2}{3}}+10 \frac{-3}{S t}\right)}$

where $S c$ is the Schmidt number which accounts for Brownian motion of very small particles. $S c$ is calculated as $S c=v / D, v$ is the kinematic viscosity of air and $D$ is the molecular diffusivity. St is the Stokes number which accounts for inertial impaction for larger size particles. $u^{*}$ is the ERA-40 wind speed at the lowest model level.

\subsubsection{Wet deposition}

Dust is removed from the atmosphere by sub-cloud scavenging. The amount of mass removed is proportional to the precipitation rate and the scavenging coefficient such that,

$C_{t}=C_{0} \exp ^{-\Lambda t}$

$C_{0}$ is the initial tracer mass $(\mathrm{kg})$ and $t$ is the model time step which is one hour. $\Lambda$ is the scavenging coefficient which has units of $\mathrm{h}^{-1}$ (Seinfeld and Pandis, 1998). The scavenging coefficient is calculated using the following empirical relationship (Brandt et al., 2002).

$\Lambda=A p_{\mathrm{Z}}^{B}$

where $A=8.4 \times 10^{-5}$ and $B=0.79$ for both convective and large scale precipitation. $p_{\mathrm{z}}$ is the large scale or convective precipitation rate $\left(\mathrm{mm} \mathrm{h}^{-1}\right)$ at a particular height. $p_{\mathrm{z}}$ is calculated from the surface precipitation rate $\left(p_{0}\right)$ by assuming a vertical precipitation profile (Fig. 6). For large scale precipitation, the cloud is divided into an upper and a lower part. The cloud base assumed to be located at $90 \%$ of the surface pressure, the cloud middle at about $80 \%$ and the cloud top 


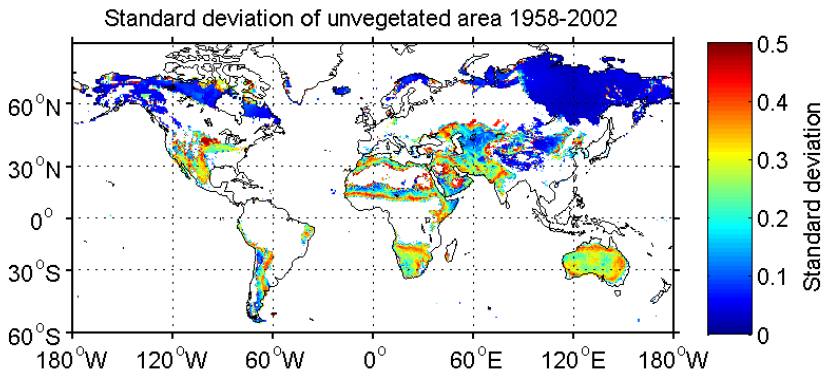

Fig. 3. Standard deviation of the un-vegetated area simulated by LPJ over the period 1958-2002.

at about $50 \%$. The precipitation varies linearly in the upper part of the cloud, from zero at cloud top, to a value $x$, at cloud middle. $x$ is calculated from the medium and low cloud amounts such that

$x=p_{0} \frac{A_{\text {med }}}{\left(A_{\text {med }}+A_{\text {low }}\right)}$

where $A_{\text {med }}$ and $A_{\text {low }}$ are the ERA-40 6 hourly low and medium cloud amounts.

For convective precipitation, the cloud base is assumed to be at a pressure which is $90 \%$ of surface pressure, and cloud top is assumed to be at the tropopause. The amount of precipitation varies linearly from zero at cloud top to the surface value at the base of the cloud. This figure has been referred to previously in the text. Alternative sub-cloud scavenging schemes are tested in Sect. 2.6.

\subsection{A baseline dust simulation}

Figure 3 shows a schematic of the dust model. A baseline simulation is run using an arbitrary choice of values for mfparlim, $\operatorname{sd}_{l i m}, \eta$ and $\mathrm{sm}_{\text {lim }}$. These parameters will be tuned in the following section. For the baseline simulation the values selected are $\operatorname{mfpar}_{\mathrm{lim}}=0.5, \mathrm{sd}_{\mathrm{lim}}=0.1 \mathrm{~m}, \eta=0.66$, $\mathrm{sm}_{\mathrm{lim}}=4 \%$. Figure 5 shows a plot of surface emissions and deposition fields. It can be seen that dry deposition is the dominant mechanism for dust removal close to the source regions owing to the abundance of heavy particles close to the source. In addition to this, there is generally a lack of precipitation in these regions which means dry deposition is the prevailing mechanism for removal. In contrast, wet deposition dominates the removal in regions far from the source. The annual mean surface emissions predicted by the un-tuned model averaged over the years $1987-1990$ is $1944 \mathrm{Mtyr}^{-1}$.

\subsection{Tuning threshold limits for surface emissions}

We decide to tune four threshold limits in the model; mfpar ${ }_{\text {lim }}, \mathrm{sd}_{\mathrm{lim}}, \eta$ and $\mathrm{sm}_{\mathrm{lim}}$. The reasons for tuning these particular set of parameters are discussed in turn below. Values for the thresholds are selected using Latin Hypercube
Sampling (McKay et al., 1979). To use this technique a sensible minimum and maximum range for each parameter and the total number of experiments must be known. To estimate the minimum and maximum range for the tunable parameters, extreme values for the threshold limits are tested. The model is run multiple times using different values for the threshold limits and emissions are compared to a simulation using the model of Tegen et al. (2002). Data from the year 1987 is used for comparison.

The first parameter to tune is the vegetation threshold mfpar $_{\text {lim. }}$. The value for this parameter is poorly constrained by observations. Where observations are available they may be limited to a particular region (Kimura et al., 2009). Often the vegetation threshold is chosen subjectively to give reasonable estimates of dust source regions. For example, studies which use BIOME3 or BIOME4 models, use an annual mean leaf area index (LAI) of 1.2 (Lunt and Valdes, 2002) or an annual maximum LAI of $0.35 \mathrm{~m}^{2} \mathrm{~m}^{-2}$ in the tropics and subtropics and $0.20 \mathrm{~m}^{2} \mathrm{~m}^{-2}$ in colder regions (Mahowald et al., 1999).

Alternatively, Zender et al. (2003) used a satellite derived vegetation dataset a single threshold of $0.3 \mathrm{~m}^{2} \mathrm{~m}^{-2}$. Another approach has been to use different thresholds depending on the vegetation type. Tegen et al. (2002) used a monthly FPAR limit of 0.25 for grasses and annual mean FPAR of 0.5 for shrubs which was derived from NDVI observations.

We decided to tune the mfpar ${ }_{\text {lim }}$ for LPJ within a minimum and maximum range of $0.2-0.5$. A comparison with the model of Tegen et al. (2002) for the same period shows that choosing values lower than 0.2 leads to very little dust emissions in South America, North America, South Africa and Australia. Choosing an mfpar $_{\text {lim }}$ threshold greater than 0.5 leads to dust emissions from highly productive grass lands where $\mathrm{C} 4$ grass is present.

The second parameter tuned is the soil moisture threshold $\mathrm{sd}_{\text {lim }}$. Dust models treat the interaction between soil moisture and dust emissions in a different ways. Lunt and Valdes (2002) assumed that dust emissions occur when $\mathrm{sm}_{\mathrm{lim}}$ is less than $10 \%$ by volume. Tegen et al. (2002) allowed dust emissions whenever the soil was not at field capacity. Werner et al. (2002) allowed dust emissions if the relative soil moisture over the total soil depth was less than $1 \%$. Other models use surface wetness limit of 0.5 (Ginoux et al., 2001) or 0.4 (Yue et al., 2009). Alternatively, some models circumvent using a soil moisture limit by assuming no emissions occur when there are consecutive days with no precipitation (Grini et al., 2005; Myhre et al., 2003; Claquin et al., 1999). One of the reasons to tune $\mathrm{sm}_{\mathrm{lim}}$ is because wind speeds can dry the soil surface causing emissions even though the soil beneath may be at field capacity. Furthermore, in remote regions, uncertainty in the precipitation rates, caused by the lack of observational data, means that the soil moisture may not be known well. The $\mathrm{sm}_{\mathrm{lim}}$ values are selected within the range of $2 \%$ to $5 \%$. Values lower than $2 \%$ leads to an under prediction of dust emissions from central Asia, Australia and North 


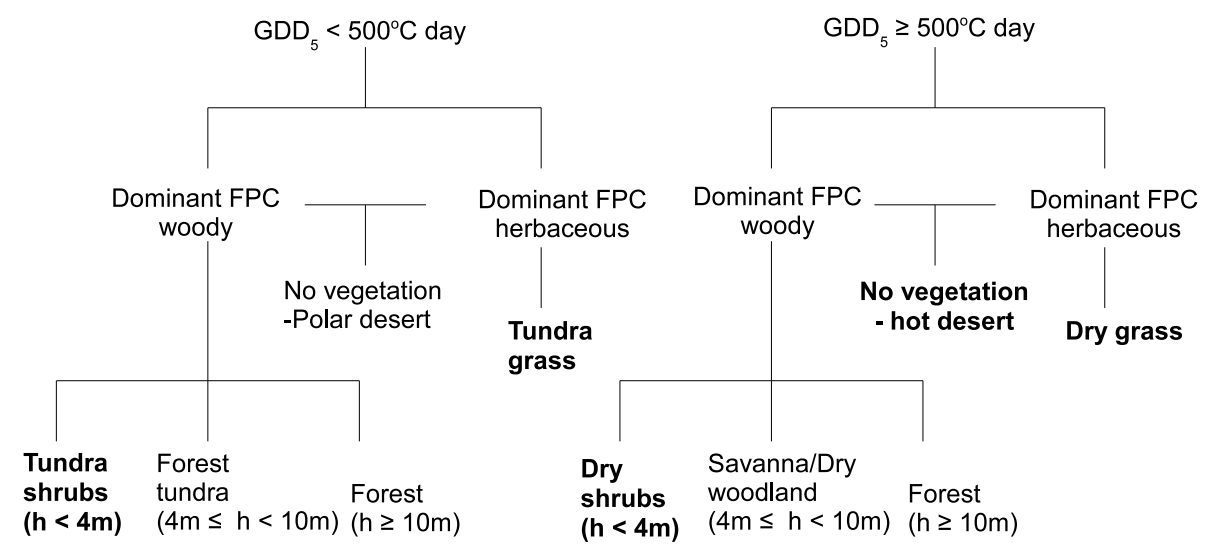

Fig. 4. Scheme used to calculate biomes from LPJ annual mean FPC, GDD 5 and tree height. The scheme has been adapted from Joos et al. (2004). Dust emitting biomes are designated in bold.

America. The upper bound was selected so as to include emissions from the boundaries of the deserts, for example in the Sahel in North Africa.

The third parameter tuned is the threshold limit for snow depth $\operatorname{sd}_{\mathrm{lim}}$. This threshold represents the snow depth at which a model grid box is completely covered by snow. We expect the choice of value for $\operatorname{sd}_{\text {lim }}$ to affect surface emissions at high latitudes but to have a relatively small impact on the total global dust loading. Typical values used in previous studies have been $0.05 \mathrm{~m}$ (Zender et al., 2003; Werner et al., 2002) and $0.015 \mathrm{~m}$ (Tegen et al., 2002). sd lim threshold limits are tuned within the a range of $0.01 \mathrm{~m}$ to $0.1 \mathrm{~m}$. Choosing a threshold greater $0.1 \mathrm{~m}$ gives rise to an abundance of dust emissions at high latitudes in winter while choosing a threshold smaller than $0.01 \mathrm{~m}$ eliminates dust emissions from the Gobi Desert.

The fourth parameter tuned is $\eta$ from Eq. (11). This parameter increases or decrease $u_{t}^{*}$ for each particle by a constant factor, while retaining the same form of size dependence between $u_{t}^{*}$ and the particle diameter as calculated by Iversen and White (1982). This parameter is tuned to account for uncertainties in the properties of the surface such as surface crusting or cultivation which is not parameterised in the model. Tegen et al. (2002) used a value of 0.66 for $\eta$. We tune $\eta$ range over a range of $0.4-1$. Choosing a value of 0.4 for the $\eta$ gives annual mean dust emissions of $3000 \mathrm{Mtyr}^{-1}$ which is the upper estimate predicted by other dust modeling studies (Tegen and Fung, 1994; Mahowald et al., 1999). Choosing a value of 1 for the $\eta$ means the threshold friction velocities are un-scaled. This results in very low annual mean dust emissions of $60 \mathrm{Mtyr}^{-1}$.

21 sets of surface emissions are generated for the years 1987 to 1990 . This comprises of 20 sets of surface emissions calculated using Latin Hypercube Sampling and the untuned emissions from the baseline simulation. The surface emissions are combined with three sub-cloud scavenging schemes (see Sect. 2.6). Each set of surface emissions con- tains 8 tracers resulting in 504 sets of experiments. Running this number of experiments provides a balance between computational expense and coverage of parameter space.

\subsection{Sub-cloud scavenging schemes}

The un-tuned model uses a sub-cloud scavenging scheme which is independent of the size of the precipitating cloud droplets (Brandt et al., 2002). We test another possible subcloud scavenging scheme, in which the scavenging coefficient is calculated as a function of the cloud droplet size. The parameterisation is based on the semi-empirical expression for the aerosol droplet collision efficiency described by Slinn (1983). The collision efficiency is calculated as a function of particle size as,

$$
\begin{aligned}
E= & \frac{4}{\operatorname{ReSc}}\left[1+0.4 \operatorname{Re}^{\frac{1}{2}} S c^{\frac{1}{3}}+0.16 \operatorname{Re}^{\frac{1}{2}} S c^{\frac{1}{2}}\right] \\
& +4 \phi\left[\omega^{-1}+\left(1+2 \operatorname{Re} e^{\frac{1}{2}}\right) \phi\right]+\left(\frac{S t-S}{S t-S+\frac{2}{3}}\right)^{\frac{3}{2}}
\end{aligned}
$$

(Seinfeld, 1998). Re is the Reynolds number, $S c$ is the Schmidt number, $S t$ is the Stokes number, $\varphi$ is the ratio of the particle diameter to the drop diameter, $\omega$ is the ratio of the water viscosity to air viscosity and

$$
S=\frac{1.2+\frac{1}{12} \ln (1+R e)}{1+\ln (1+R e)}
$$

The scavenging coefficient is calculated from the collision coefficient by assuming a monotonic rain droplet diameter,

$\Lambda=\frac{3}{2} \frac{E p_{\mathrm{z}}}{D_{\text {droplet }}}$

where $D_{\text {droplet }}$ is the rain droplet size $(\mathrm{mm})$ and $p_{\mathrm{z}}$ is the precipitation rate $\left(\mathrm{mm} \mathrm{h}^{-1}\right) . \Lambda$ is calculated for a rain droplet with diameter $0.5 \mathrm{~mm}$ and $0.1 \mathrm{~mm}$. 

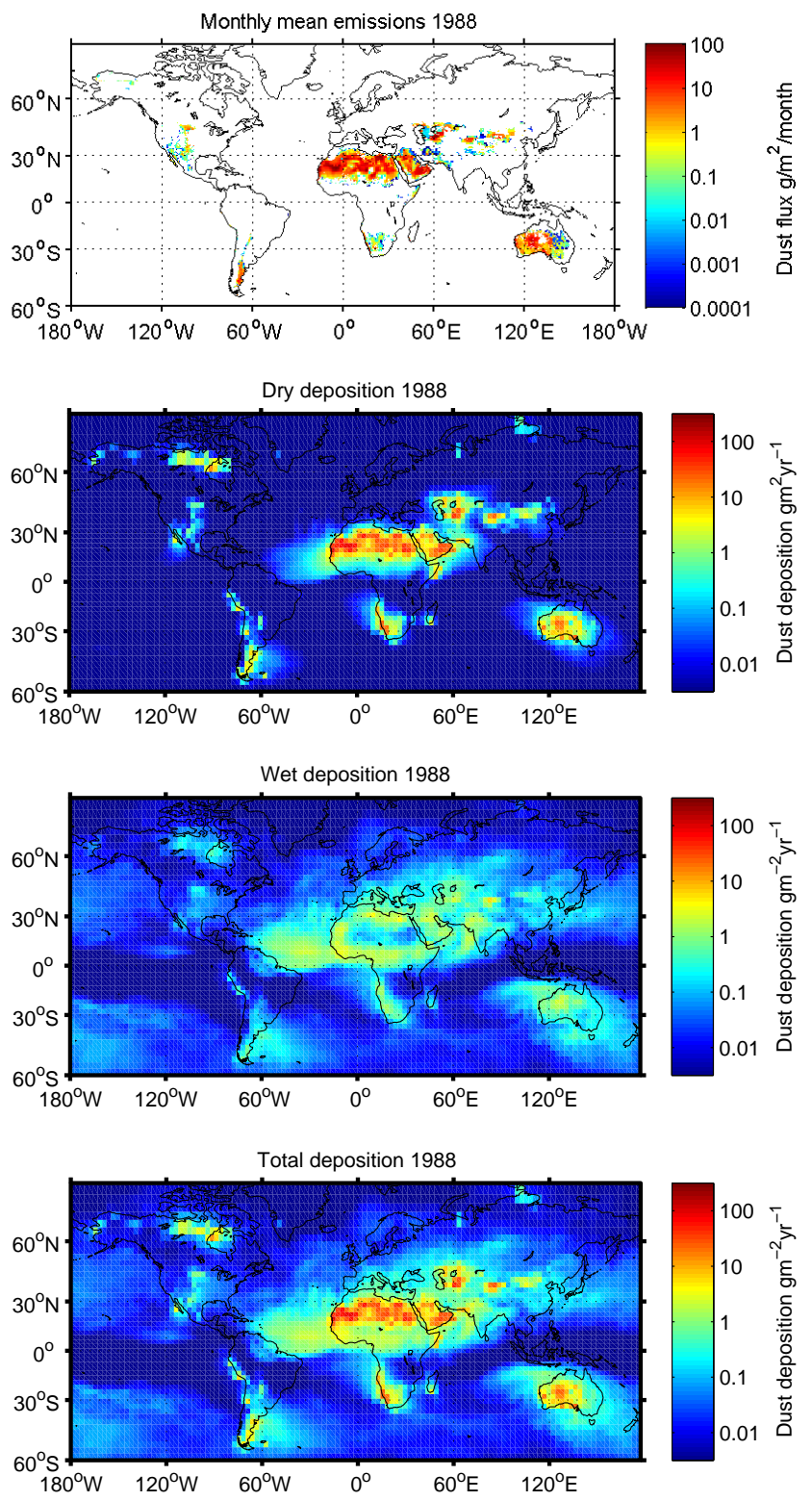

Fig. 5. Emissions and deposition fields from the baseline dust simulation.

Figure 7 shows the scavenging coefficient calculated for the three schemes using a precipitation rate of $1 \mathrm{~mm} \mathrm{~h}^{-1}$. The straight line corresponds to the particle size independent sub-cloud scavenging scheme used in the un-tuned model. The particle size dependent removal schemes have a hook shaped curve which indicates that scavenging is efficient for very small and very large particles. For very large particles the process of inertial impact dominates the removal while Brownian diffusion is important for very small particles. However, for particles in the region of $0.1 \mu \mathrm{m}$ diameter scavenging is not as efficient. The simulations are run for the years 1987-1990 and amount of dust removed by wet and dry deposition and the surface concentrations are output daily.

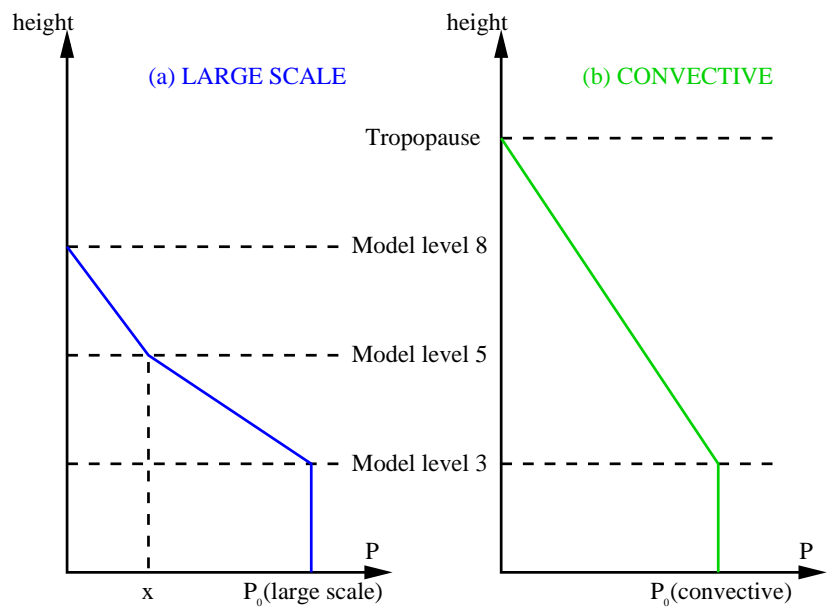

Fig. 6. Calculation of 3-D precipitation rates from surface precipitation rates by assuming a vertical cloud profile based on low and medium cloud fractional cloud amounts. The scheme is taken from Lunt (2001).

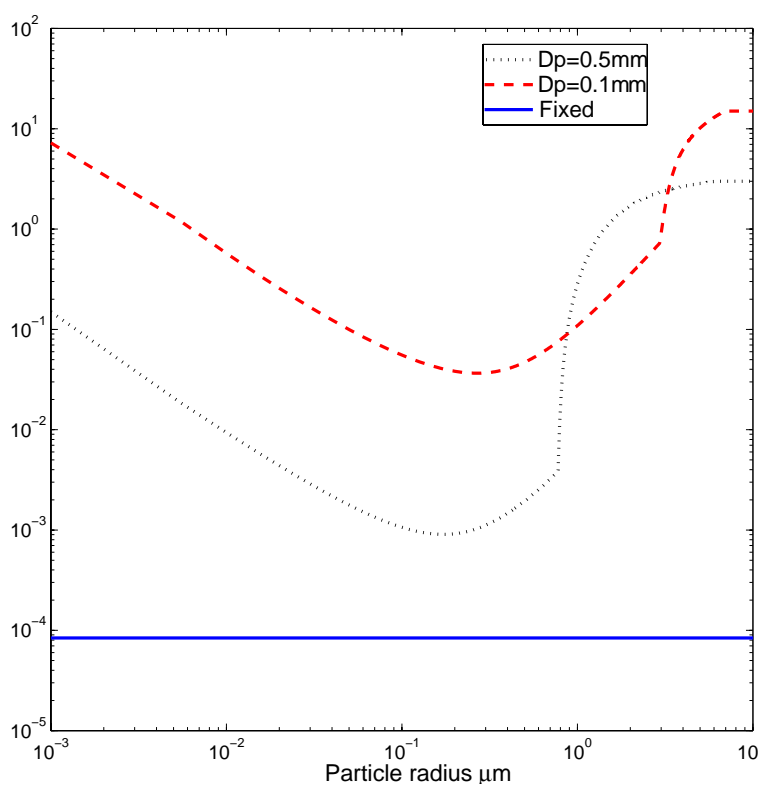

Fig. 7. Comparison between the scavenging coefficients for three different wet deposition schemes. The dashed lines correspond to the size dependent removal schemes (Slinn, 1983) while the fixed line corresponds to the size independent removal scheme (Brandt et al., 2002). A precipitation rate of $1 \mathrm{~mm} \mathrm{~h}^{-1}$ is used to calculate the scavenging coefficient for this figure.

\subsection{Target datasets}

Three measurement datasets are used to evaluate the performance of the experiments. The first is dust deposition rates to the ocean from marine sediment traps from the Dust Indicators and Records of Terrestrial and MArine Palaeoenvironments dataset (Kohfeld and Harrison, 2001). This data has 
been compiled by Tegen et al. (2002) and has been filtered to only include observations taken for more than 50 days. Sites where observations could potentially be contaminated by fluvial inputs or hemipelagic reworking have been removed. In regions where multiple observations occur within one model grid box the mean deposition rate is used. Deposition rates obtained from ice cores, marine cores and loess are excluded as these observations represent dust deposition over many hundreds of years and are not comparable to the relatively short tuning period.

The second dataset consists of dust deposition rates compiled by Ginoux et al. (2001). This dataset set contains observations made in the Pacific Ocean and from high resolution ice core records. Observations which do not occur duing the 1980s and 1990s are excluded in the analysis.

The third dataset comprises of surface concentration measurements from the University of Miami aerosol network (provided by J. Prospero and D. Savoie). The network contains observations of monthly surface concentrations made at sites during the 1980s and 1990s. The annual mean surface concentrations are calculated from monthly data and used in the analysis. Figure 8 shows the spatial distribution of the DIRTMAP deposition, Ginoux deposition and University of Miami surface concentration observations.

\subsection{Results}

To evaluate the best experiment in the ensemble, a skill score is used. The skills score is based on the normalised root mean square error (NRMSE) between the observations and the model data. The NRMSE is calculated as

$\mathrm{NRMSE}=\sqrt{\frac{\mathrm{MSE}}{\sigma^{2}}}$

where $\sigma^{2}$ is the variance of the observations and MSE is the mean square error.

$\sigma^{2}$ is calculated from

$\sigma^{2}=\frac{\sum_{i=1}^{n}\left(o_{i}-\mu\right)^{2}}{n}$

where $o_{i}$ is the observed data, $\mu$ is the mean of the observations and $n$ is the number of observations.

The MSE is

$\mathrm{MSE}=\frac{\sum_{i=1}^{n}\left(m_{i}-o_{i}\right)^{2}}{n}$

where $m_{i}$ is the modelled data.

Prior to calculating the NRMSE, a global tuning factor is calculated $(T)$. This is the value by which the data is adjusted by to minimize the NRMSE. $T$ acts to move the modeled data up or down so that it fits on the ideal 1:1 line with the least amount of scatter. The total error $(Q)$ is calculated from the NRMSE such that,

$Q=\sum_{j=1}^{3} w_{j} \mathrm{NRMSE}_{j}$

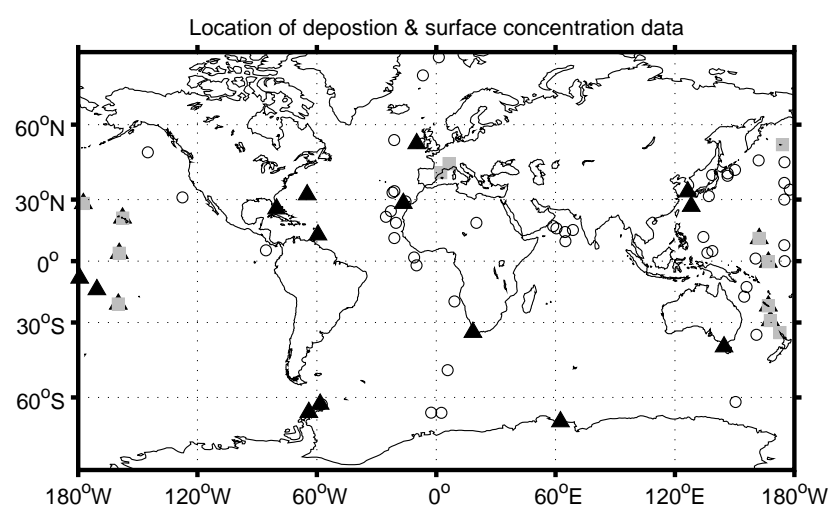

Fig. 8. Location of DIRTMAP sites (circles), Ginoux deposition (squares) and University of Miami data (triangles).

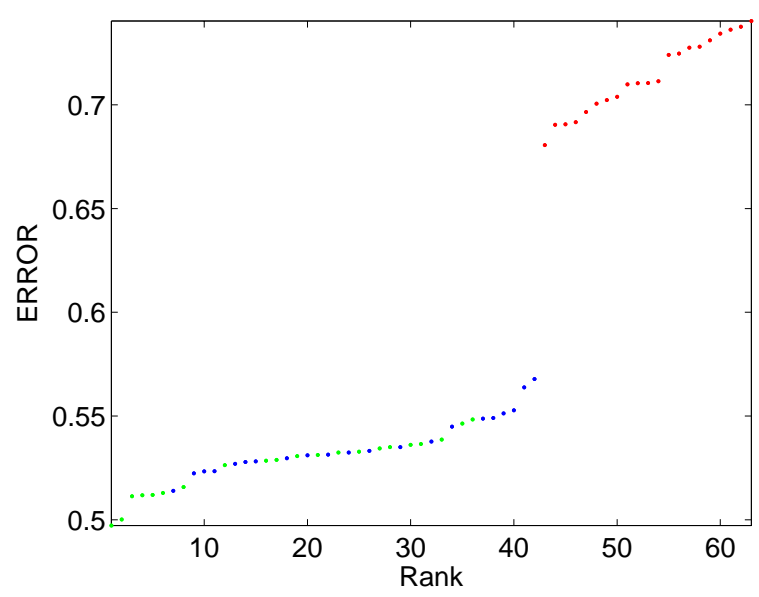

Fig. 9. Model error plotted against position of experiment in the ensemble, Brandt removal (green), Slinn removal $D_{\mathrm{p}}=0.1 \mathrm{~mm}$ (blue) and Slinn removal $D_{\mathrm{p}}=0.5 \mathrm{~mm}$ (red).

$w_{j}$ is a weighting factor for each dataset. Because there are two deposition datasets each is weighted half as much as the surface concentration data. A similar weighting approach has been used by Cakmur et al. (2006) who used multiple observational datasets to contrain dust emissions. Weighting each dataset evenly does not change the outcome for the best and worst experiment but changes the order of the experiments within the ensemble.

Table 2 lists the experiments ranked according to the total error $Q$. The threshold values for each experiment, the removal scheme and the $T$ values for each dataset are listed in the table. The top five best experiments use the Brandt removal scheme and a soil moisture threshold of $2 \%$. There is no area of parameter space which results in high skills scores for the other threshold parameters.

Figure 9 shows the model error plotted against the position of experiment in the ensemble. Experiments which use the Brandt removal scheme and the Slinn removal scheme with 
Table 2. Tuning experiments ranked according to the total error. Threshold limits used to determine surface emissions, the tuning factor $\mathrm{T}$ and the removal schemes are also listed.

\begin{tabular}{|c|c|c|c|c|c|c|c|c|c|}
\hline Expt ID & Removal Scheme & mfpar $_{\text {lim }}$ & $\mathrm{sm}_{\lim }$ & $\operatorname{sd}_{\text {lim }}$ & $\eta$ & $T_{\text {Dirtmap }}$ & $T_{\text {Ginoux }}$ & $T_{\text {Miami }}$ & $Q$ \\
\hline 54 & Brandt & 0.33 & 0.02 & 0.08 & 0.90 & 7.26 & 10.54 & 7.61 & 0.50 \\
\hline 44 & Brandt & 0.37 & 0.02 & 0.10 & 0.55 & 0.21 & 0.89 & 0.77 & 0.50 \\
\hline 45 & Brandt & 0.23 & 0.02 & 0.05 & 0.80 & 2.04 & 4.22 & 3.01 & 0.51 \\
\hline 52 & Brandt & 0.28 & 0.02 & 0.07 & 0.58 & 0.23 & 0.87 & 0.69 & 0.51 \\
\hline 62 & Brandt & 0.43 & 0.02 & 0.03 & 0.82 & 2.94 & 5.74 & 4.22 & 0.51 \\
\hline 46 & Brandt & 0.32 & 0.03 & 0.07 & 0.93 & 9.38 & 11.93 & 7.83 & 0.51 \\
\hline 25 & Slinn droplet $=0.1 \mathrm{~mm}$ & 0.32 & 0.03 & 0.07 & 0.93 & 10.42 & 12.74 & 5.18 & 0.51 \\
\hline 63 & Brandt & 0.27 & 0.02 & 0.09 & 0.64 & 0.37 & 1.27 & 0.95 & 0.52 \\
\hline 24 & Slinn droplet $=0.1 \mathrm{~mm}$ & 0.23 & 0.02 & 0.05 & 0.80 & 2.07 & 4.85 & 1.97 & 0.52 \\
\hline 29 & Slinn droplet $=0.1 \mathrm{~mm}$ & 0.30 & 0.04 & 0.02 & 0.99 & 19.58 & 20.46 & 8.40 & 0.52 \\
\hline 34 & Slinn droplet $=0.1 \mathrm{~mm}$ & 0.25 & 0.05 & 0.03 & 0.73 & 0.97 & 2.45 & 0.97 & 0.52 \\
\hline 59 & Brandt & 0.40 & 0.03 & 0.04 & 0.78 & 1.57 & 3.14 & 2.08 & 0.53 \\
\hline 33 & Slinn droplet $=0.1 \mathrm{~mm}$ & 0.33 & 0.02 & 0.08 & 0.90 & 7.77 & 11.51 & 4.77 & 0.53 \\
\hline 38 & Slinn droplet $=0.1 \mathrm{~mm}$ & 0.40 & 0.03 & 0.04 & 0.78 & 1.60 & 3.40 & 1.41 & 0.53 \\
\hline 35 & Slinn droplet $=0.1 \mathrm{~mm}$ & 0.24 & 0.03 & 0.04 & 0.60 & 0.26 & 1.02 & 0.43 & 0.53 \\
\hline 56 & Brandt & 0.24 & 0.03 & 0.04 & 0.60 & 0.26 & 0.89 & 0.63 & 0.53 \\
\hline 48 & Brandt & 0.46 & 0.03 & 0.06 & 0.41 & 0.11 & 0.31 & 0.23 & 0.53 \\
\hline 28 & Slinn droplet $=0.1 \mathrm{~mm}$ & 0.36 & 0.04 & 0.05 & 0.71 & 0.73 & 1.98 & 0.83 & 0.53 \\
\hline 49 & Brandt & 0.36 & 0.04 & 0.05 & 0.71 & 0.73 & 1.83 & 1.22 & 0.53 \\
\hline 42 & Slinn droplet $=0.1 \mathrm{~mm}$ & 0.27 & 0.02 & 0.09 & 0.64 & 0.37 & 1.45 & 0.62 & 0.53 \\
\hline 57 & Brandt & 0.49 & 0.02 & 0.00 & 0.68 & 0.52 & 1.62 & 1.27 & 0.53 \\
\hline 26 & Slinn droplet $=0.1 \mathrm{~mm}$ & 0.21 & 0.04 & 0.02 & 0.46 & 0.13 & 0.49 & 0.20 & 0.53 \\
\hline 50 & Brandt & 0.30 & 0.04 & 0.02 & 0.99 & 16.95 & 19.96 & 12.54 & 0.53 \\
\hline 39 & Slinn droplet $=0.1 \mathrm{~mm}$ & 0.41 & 0.03 & 0.08 & 0.96 & 13.88 & 14.12 & 5.99 & 0.53 \\
\hline 60 & Brandt & 0.41 & 0.03 & 0.08 & 0.96 & 12.15 & 14.12 & 9.24 & 0.53 \\
\hline 40 & Slinn droplet $=0.1 \mathrm{~mm}$ & 0.44 & 0.04 & 0.09 & 0.87 & 4.97 & 6.11 & 2.55 & 0.53 \\
\hline 47 & Brandt & 0.21 & 0.04 & 0.02 & 0.46 & 0.13 & 0.43 & 0.30 & 0.53 \\
\hline 55 & Brandt & 0.25 & 0.05 & 0.03 & 0.73 & 0.96 & 2.23 & 1.38 & 0.54 \\
\hline 37 & Slinn droplet $=0.1 \mathrm{~mm}$ & 0.35 & 0.04 & 0.01 & 0.66 & 0.42 & 1.36 & 0.58 & 0.54 \\
\hline 58 & Brandt & 0.35 & 0.04 & 0.01 & 0.66 & 0.42 & 1.24 & 0.84 & 0.54 \\
\hline 51 & Brandt & 0.39 & 0.05 & 0.01 & 0.46 & 0.17 & 0.38 & 0.27 & 0.54 \\
\hline 31 & Slinn droplet $=0.1 \mathrm{~mm}$ & 0.28 & 0.02 & 0.07 & 0.58 & 0.23 & 0.99 & 0.44 & 0.54 \\
\hline 61 & Brandt & 0.44 & 0.04 & 0.09 & 0.87 & 4.60 & 6.09 & 3.84 & 0.54 \\
\hline 30 & Slinn droplet $=0.1 \mathrm{~mm}$ & 0.39 & 0.05 & 0.01 & 0.46 & 0.12 & 0.42 & 0.18 & 0.54 \\
\hline 53 & Brandt & 0.47 & 0.05 & 0.06 & 0.51 & 0.16 & 0.51 & 0.36 & 0.55 \\
\hline 43 & Brandt & 0.50 & 0.04 & 0.01 & 0.66 & 0.44 & 1.22 & 0.84 & 0.55 \\
\hline 27 & Slinn droplet $=0.1 \mathrm{~mm}$ & 0.46 & 0.03 & 0.06 & 0.41 & 0.11 & 0.33 & 0.15 & 0.55 \\
\hline 41 & Slinn droplet $=0.1 \mathrm{~mm}$ & 0.43 & 0.02 & 0.03 & 0.82 & 3.00 & 6.21 & 2.63 & 0.55 \\
\hline 22 & Slinn droplet $=0.1 \mathrm{~mm}$ & 0.50 & 0.04 & 0.01 & 0.66 & 0.44 & 1.28 & 0.56 & 0.55 \\
\hline 32 & Slinn droplet $=0.1 \mathrm{~mm}$ & 0.47 & 0.05 & 0.06 & 0.51 & 0.16 & 0.53 & 0.24 & 0.55 \\
\hline 36 & Slinn droplet $=0.1 \mathrm{~mm}$ & 0.49 & 0.02 & 0.00 & 0.68 & 0.52 & 1.78 & 0.80 & 0.56 \\
\hline 23 & Slinn droplet $=0.1 \mathrm{~mm}$ & 0.37 & 0.02 & 0.10 & 0.55 & 0.20 & 0.99 & 0.45 & 0.57 \\
\hline 3 & Slinn droplet $=0.5 \mathrm{~mm}$ & 0.23 & 0.02 & 0.05 & 0.80 & 2.05 & 4.37 & 0.71 & 0.68 \\
\hline 13 & Slinn droplet $=0.5 \mathrm{~mm}$ & 0.25 & 0.05 & 0.03 & 0.73 & 0.96 & 2.31 & 0.34 & 0.69 \\
\hline 5 & Slinn droplet $=0.5 \mathrm{~mm}$ & 0.21 & 0.04 & 0.02 & 0.46 & 0.13 & 0.45 & 0.07 & 0.69 \\
\hline 14 & Slinn droplet $=0.5 \mathrm{~mm}$ & 0.24 & 0.03 & 0.04 & 0.60 & 0.26 & 0.93 & 0.15 & 0.69 \\
\hline 21 & Slinn droplet $=0.5 \mathrm{~mm}$ & 0.27 & 0.02 & 0.09 & 0.64 & 0.37 & 1.32 & 0.21 & 0.70 \\
\hline 4 & Slinn droplet $=0.5 \mathrm{~mm}$ & 0.32 & 0.03 & 0.07 & 0.93 & 9.92 & 12.20 & 1.68 & 0.70 \\
\hline 10 & Slinn droplet $=0.5 \mathrm{~mm}$ & 0.28 & 0.02 & 0.07 & 0.58 & 0.23 & 0.90 & 0.14 & 0.70 \\
\hline 12 & Slinn droplet $=0.5 \mathrm{~mm}$ & 0.33 & 0.02 & 0.08 & 0.90 & 7.52 & 10.72 & 1.54 & 0.70 \\
\hline 16 & Slinn droplet $=0.5 \mathrm{~mm}$ & 0.35 & 0.04 & 0.01 & 0.66 & 0.42 & 1.29 & 0.19 & 0.71 \\
\hline 17 & Slinn droplet $=0.5 \mathrm{~mm}$ & 0.40 & 0.03 & 0.04 & 0.78 & 1.58 & 3.22 & 0.45 & 0.71 \\
\hline 7 & Slinn droplet $=0.5 \mathrm{~mm}$ & 0.36 & 0.04 & 0.05 & 0.71 & 0.73 & 1.88 & 0.27 & 0.71 \\
\hline 8 & Slinn droplet $=0.5 \mathrm{~mm}$ & 0.30 & 0.04 & 0.02 & 0.99 & 18.42 & 20.46 & 2.69 & 0.71 \\
\hline 9 & Slinn droplet $=0.5 \mathrm{~mm}$ & 0.39 & 0.05 & 0.01 & 0.46 & 0.12 & 0.40 & 0.06 & 0.72 \\
\hline
\end{tabular}


Table 2. Continued.

\begin{tabular}{llllllrrrr}
\hline Expt ID & Removal Scheme & mfpar $_{\text {lim }}$ & $\mathrm{sm}_{\lim }$ & $\mathrm{sd}_{\lim }$ & $\eta$ & $T_{\text {Dirtmap }}$ & $T_{\text {Ginoux }}$ & $T_{\text {Miami }}$ & $Q$ \\
\hline 20 & Slinn droplet $=0.5 \mathrm{~mm}$ & 0.43 & 0.02 & 0.03 & 0.82 & 2.97 & 5.84 & 0.81 & 0.72 \\
19 & Slinn droplet $=0.5 \mathrm{~mm}$ & 0.44 & 0.04 & 0.09 & 0.87 & 4.78 & 6.17 & 0.75 & 0.73 \\
2 & Slinn droplet $=0.5 \mathrm{~mm}$ & 0.37 & 0.02 & 0.10 & 0.55 & 0.20 & 0.92 & 0.14 & 0.73 \\
18 & Slinn droplet $=0.5 \mathrm{~mm}$ & 0.41 & 0.03 & 0.08 & 0.96 & 13.06 & 14.32 & 1.75 & 0.73 \\
6 & Slinn droplet $=0.5 \mathrm{~mm}$ & 0.46 & 0.03 & 0.06 & 0.41 & 0.11 & 0.32 & 0.04 & 0.73 \\
15 & Slinn droplet $=0.5 \mathrm{~mm}$ & 0.49 & 0.02 & 0.00 & 0.68 & 0.52 & 1.66 & 0.24 & 0.74 \\
1 & Slinn droplet $=0.5 \mathrm{~mm}$ & 0.50 & 0.04 & 0.01 & 0.66 & 0.44 & 1.24 & 0.16 & 0.74 \\
11 & Slinn droplet $=0.5 \mathrm{~mm}$ & 0.47 & 0.05 & 0.06 & 0.51 & 0.16 & 0.52 & 0.07 & 0.74 \\
\hline
\end{tabular}

droplet diameter $0.1 \mathrm{~mm}$ perform the best. A sharp increase in the model error can be seen for the experiments which use the $0.5 \mathrm{~mm}$ rain droplet. The experiment with the lowest total error has threshold limits ffpar $_{\lim }=0.33, \mathrm{sd}_{\mathrm{lim}}=0.08 \mathrm{~m}, \eta=$ $0.9, \mathrm{sm}_{\mathrm{lim}}=2 \%$. This results in estimates of the annual mean surface emissions which range from $1600 \mathrm{Mtyr}^{-1}(T=7.26$, DIRTMAP) and $3000 \mathrm{Mtyr}^{-1},(T=7.61$, University of Miami) and $2400 \mathrm{Mtyr}^{-1},(T=10.54$, Ginoux $)$. The mean $T$ value gives emissions of $1900 \mathrm{Mtyr}^{-1}$. It is not expected that the value for $T$ be exactly the same for each dataset. This is because the observations contain inter-annual varibility and different degrees of measurement error. There are also differences in the spatial distribution of the observations. For example, many of the University of Miami sites are located far from the source region, while the DIRTMAP data contains more sites downwind of the source regions.

Figure 10 shows a comparison between the model data and the observations for the best and the worst performing experiment in the ensemble. The best experiment shows improved estimates of deposition rates and surface concentrations to the North and South Pacific, Arabian Sea and the North Atlantic and the Southern Atlantic.

Experiments are listed according to their NRMSE with each dataset separately in Tables 3, 4 and 5. The comparison with the DIRTMAP data shows the best three experiments have the same threshold limits but use different removal schemes. This is because the DIRTMAP data contains sites close to the source region, and thus show more sensitivity to the choice of threshold limits and less sensitivity to the removal scheme. The comparison with the surface concentrations and Ginoux deposition data, show that the Slinn removal scheme with droplet diameter $0.1 \mathrm{~mm}$ and the Brandt removal scheme produce high skills scores.

\section{Conclusions}

This work has described how the LPJ dynamic global vegetation model has been used to simulate the temporal variability in vegetation cover within the framework of a dust cycle model. The development of the model has been motivated by the fact that current off-line dust models do not simulate dynamic vegetation. The model has been tuned by producing an ensemble of simulations and using a skills score to select the best performing experiment. Surface emissions which agree best with observations range from 1600 to $2400 \mathrm{Mtyr}^{-1}$. This estimate lies within the range reported by studies (Cakmur et al., 2006; Yue et al., 2009; Tanaka and Chiba, 2006; Ginoux et al., 2004). The tuning carried out explored only a small subset of the possible parametric and structural uncertainty in the model, but resulted in improved estimates of dust deposition to the North Atlantic, North Pacific, South pacific and the Arabian Sea.

The LPJ-dust model has many limitations. Currently, roughness length is assumed have a constant value for all dust emitting regions. Wind speed has a non-linear relationship with dust emissions, which means the way in which the roughness length is treated, may have a large impact on the surface emissions. One way to improve this is to parameterise the roughness length as a function of the leaf area index and the vegetation stand height simulated by LPJ using an empirical relationship such as that by Lindroth (1993).

Another limitation is that the current set of PFT in LPJ is not sufficient to characterise all the possible vegetation types. In particular, LPJ does not simulate shrub PFTs which may be important in semi-arid regions. The model assumes that short trees are shrubs even though their physiological and morphological attributes may be different to that of trees. Furthermore, the model uses the same threshold limit for both shrub and grasses to calculate dust emissions. This could be improved by using a threshold specific to different vegetation types in a similar way to Tegen et al. (2002).

Another limitation of the model, is that the temporal variability in sediment supply is not parameterised. Sediment supply is enhanced when flooding deposits fine grain material which can be easily eroded. Crusting of the surface which occurs when the soil dries out reduces the sediment supply. Zender and Kwon (2005) showed that this may be an important process and may help explain why dust models underestimate inter-annual variability. 
Table 3. Tuning experiments ranked according to NRMSE with DIRTMAP deposition rates.

\begin{tabular}{|c|c|c|c|c|c|c|c|}
\hline Expt ID & Removal Scheme & mfpar $_{\text {lim }}$ & $\mathrm{sm}_{\lim }$ & $\operatorname{sd}_{\lim }$ & $\eta$ & $T_{\text {Ginoux }}$ & NRMSE \\
\hline 40 & Slinn droplet $=0.1 \mathrm{~mm}$ & 0.44 & 0.04 & 0.09 & 0.87 & 4.97 & 1.04 \\
\hline 61 & Brandt & 0.44 & 0.04 & 0.09 & 0.87 & 4.60 & 1.04 \\
\hline 19 & Slinn droplet $=0.5 \mathrm{~mm}$ & 0.44 & 0.04 & 0.09 & 0.87 & 4.78 & 1.05 \\
\hline 25 & Slinn droplet $=0.1 \mathrm{~mm}$ & 0.32 & 0.03 & 0.07 & 0.93 & 10.42 & 1.05 \\
\hline 33 & Slinn droplet $=0.1 \mathrm{~mm}$ & 0.33 & 0.02 & 0.08 & 0.90 & 7.77 & 1.05 \\
\hline 39 & Slinn droplet $=0.1 \mathrm{~mm}$ & 0.41 & 0.03 & 0.08 & 0.96 & 13.88 & 1.05 \\
\hline 54 & Brandt & 0.33 & 0.02 & 0.08 & 0.90 & 7.26 & 1.05 \\
\hline 12 & Slinn droplet $=0.5 \mathrm{~mm}$ & 0.33 & 0.02 & 0.08 & 0.90 & 7.52 & 1.05 \\
\hline 4 & Slinn droplet $=0.5 \mathrm{~mm}$ & 0.32 & 0.03 & 0.07 & 0.93 & 9.92 & 1.06 \\
\hline 46 & Brandt & 0.32 & 0.03 & 0.07 & 0.93 & 9.38 & 1.06 \\
\hline 62 & Brandt & 0.43 & 0.02 & 0.03 & 0.82 & 2.94 & 1.06 \\
\hline 18 & Slinn droplet $=0.5 \mathrm{~mm}$ & 0.41 & 0.03 & 0.08 & 0.96 & 13.06 & 1.06 \\
\hline 20 & Slinn droplet $=0.5 \mathrm{~mm}$ & 0.43 & 0.02 & 0.03 & 0.82 & 2.97 & 1.06 \\
\hline 59 & Brandt & 0.40 & 0.03 & 0.04 & 0.78 & 1.57 & 1.07 \\
\hline 41 & Slinn droplet $=0.1 \mathrm{~mm}$ & 0.43 & 0.02 & 0.03 & 0.82 & 3.00 & 1.07 \\
\hline 60 & Brandt & 0.41 & 0.03 & 0.08 & 0.96 & 12.15 & 1.07 \\
\hline 29 & Slinn droplet $=0.1 \mathrm{~mm}$ & 0.30 & 0.04 & 0.02 & 0.99 & 19.58 & 1.07 \\
\hline 45 & Brandt & 0.23 & 0.02 & 0.05 & 0.80 & 2.04 & 1.07 \\
\hline 17 & Slinn droplet $=0.5 \mathrm{~mm}$ & 0.40 & 0.03 & 0.04 & 0.78 & 1.58 & 1.07 \\
\hline 38 & Slinn droplet $=0.1 \mathrm{~mm}$ & 0.40 & 0.03 & 0.04 & 0.78 & 1.60 & 1.07 \\
\hline 3 & Slinn droplet $=0.5 \mathrm{~mm}$ & 0.23 & 0.02 & 0.05 & 0.80 & 2.05 & 1.07 \\
\hline 24 & Slinn droplet $=0.1 \mathrm{~mm}$ & 0.23 & 0.02 & 0.05 & 0.80 & 2.07 & 1.07 \\
\hline 55 & Brandt & 0.25 & 0.05 & 0.03 & 0.73 & 0.96 & 1.07 \\
\hline 8 & Slinn droplet $=0.5 \mathrm{~mm}$ & 0.30 & 0.04 & 0.02 & 0.99 & 18.42 & 1.08 \\
\hline 13 & Slinn droplet $=0.5 \mathrm{~mm}$ & 0.25 & 0.05 & 0.03 & 0.73 & 0.96 & 1.08 \\
\hline 51 & Brandt & 0.39 & 0.05 & 0.01 & 0.46 & 0.17 & 1.08 \\
\hline 50 & Brandt & 0.30 & 0.04 & 0.02 & 0.99 & 16.95 & 1.08 \\
\hline 34 & Slinn droplet $=0.1 \mathrm{~mm}$ & 0.25 & 0.05 & 0.03 & 0.73 & 0.97 & 1.08 \\
\hline 49 & Brandt & 0.36 & 0.04 & 0.05 & 0.71 & 0.73 & 1.08 \\
\hline 7 & Slinn droplet $=0.5 \mathrm{~mm}$ & 0.36 & 0.04 & 0.05 & 0.71 & 0.73 & 1.08 \\
\hline 28 & Slinn droplet $=0.1 \mathrm{~mm}$ & 0.36 & 0.04 & 0.05 & 0.71 & 0.73 & 1.08 \\
\hline 43 & Brandt & 0.50 & 0.04 & 0.01 & 0.66 & 0.44 & 1.09 \\
\hline 58 & Brandt & 0.35 & 0.04 & 0.01 & 0.66 & 0.42 & 1.09 \\
\hline 57 & Brandt & 0.49 & 0.02 & 0.00 & 0.68 & 0.52 & 1.09 \\
\hline 1 & Slinn droplet $=0.5 \mathrm{~mm}$ & 0.50 & 0.04 & 0.01 & 0.66 & 0.44 & 1.09 \\
\hline 22 & Slinn droplet $=0.1 \mathrm{~mm}$ & 0.50 & 0.04 & 0.01 & 0.66 & 0.44 & 1.09 \\
\hline 48 & Brandt & 0.46 & 0.03 & 0.06 & 0.41 & 0.11 & 1.09 \\
\hline 16 & Slinn droplet $=0.5 \mathrm{~mm}$ & 0.35 & 0.04 & 0.01 & 0.66 & 0.42 & 1.09 \\
\hline 15 & Slinn droplet $=0.5 \mathrm{~mm}$ & 0.49 & 0.02 & 0.00 & 0.68 & 0.52 & 1.09 \\
\hline 37 & Slinn droplet $=0.1 \mathrm{~mm}$ & 0.35 & 0.04 & 0.01 & 0.66 & 0.42 & 1.09 \\
\hline 53 & Brandt & 0.47 & 0.05 & 0.06 & 0.51 & 0.16 & 1.09 \\
\hline 36 & Slinn droplet $=0.1 \mathrm{~mm}$ & 0.49 & 0.02 & 0.00 & 0.68 & 0.52 & 1.10 \\
\hline 6 & Slinn droplet $=0.5 \mathrm{~mm}$ & 0.46 & 0.03 & 0.06 & 0.41 & 0.11 & 1.10 \\
\hline 63 & Brandt & 0.27 & 0.02 & 0.09 & 0.64 & 0.37 & 1.10 \\
\hline 27 & Slinn droplet $=0.1 \mathrm{~mm}$ & 0.46 & 0.03 & 0.06 & 0.41 & 0.11 & 1.10 \\
\hline 56 & Brandt & 0.24 & 0.03 & 0.04 & 0.60 & 0.26 & 1.10 \\
\hline 11 & Slinn droplet $=0.5 \mathrm{~mm}$ & 0.47 & 0.05 & 0.06 & 0.51 & 0.16 & 1.10 \\
\hline 47 & Brandt & 0.21 & 0.04 & 0.02 & 0.46 & 0.13 & 1.10 \\
\hline 9 & Slinn droplet $=0.5 \mathrm{~mm}$ & 0.39 & 0.05 & 0.01 & 0.46 & 0.12 & 1.10 \\
\hline 32 & Slinn droplet $=0.1 \mathrm{~mm}$ & 0.47 & 0.05 & 0.06 & 0.51 & 0.16 & 1.10 \\
\hline 30 & Slinn droplet $=0.1 \mathrm{~mm}$ & 0.39 & 0.05 & 0.01 & 0.46 & 0.12 & 1.10 \\
\hline 21 & Slinn droplet $=0.5 \mathrm{~mm}$ & 0.27 & 0.02 & 0.09 & 0.64 & 0.37 & 1.10 \\
\hline 42 & Slinn droplet $=0.1 \mathrm{~mm}$ & 0.27 & 0.02 & 0.09 & 0.64 & 0.37 & 1.10 \\
\hline 52 & Brandt & 0.28 & 0.02 & 0.07 & 0.58 & 0.23 & 1.10 \\
\hline 14 & Slinn droplet $=0.5 \mathrm{~mm}$ & 0.24 & 0.03 & 0.04 & 0.60 & 0.26 & 1.10 \\
\hline 5 & Slinn droplet $=0.5 \mathrm{~mm}$ & 0.21 & 0.04 & 0.02 & 0.46 & 0.13 & 1.10 \\
\hline
\end{tabular}


Table 3. Continued.

\begin{tabular}{llllllll}
\hline Expt ID & Removal Scheme & mfpar $_{\text {lim }}$ & $\mathrm{sm}_{\lim }$ & $\mathrm{sd}_{\lim }$ & $\eta$ & $T_{\text {Ginoux }}$ & NRMSE \\
\hline 35 & Slinn droplet $=0.1 \mathrm{~mm}$ & 0.24 & 0.03 & 0.04 & 0.60 & 0.26 & 1.10 \\
26 & Slinn droplet $=0.1 \mathrm{~mm}$ & 0.21 & 0.04 & 0.02 & 0.46 & 0.13 & 1.10 \\
10 & Slinn droplet $=0.5 \mathrm{~mm}$ & 0.28 & 0.02 & 0.07 & 0.58 & 0.23 & 1.10 \\
44 & Brandt & 0.37 & 0.02 & 0.10 & 0.55 & 0.21 & 1.10 \\
31 & Slinn droplet $=0.1 \mathrm{~mm}$ & 0.28 & 0.02 & 0.07 & 0.58 & 0.23 & 1.11 \\
2 & Slinn droplet $=0.5 \mathrm{~mm}$ & 0.37 & 0.02 & 0.10 & 0.55 & 0.20 & 1.11 \\
23 & Slinn droplet $=0.1 \mathrm{~mm}$ & 0.37 & 0.02 & 0.10 & 0.55 & 0.20 & 1.11 \\
\hline
\end{tabular}

Table 4. Tuning experiments ranked according to NRMSE with Ginoux deposition rates.

\begin{tabular}{|c|c|c|c|c|c|c|c|}
\hline Expt ID & Removal Scheme & mfpar $_{\text {lim }}$ & $\mathrm{sm}_{\lim }$ & $\operatorname{sd}_{\text {lim }}$ & $\eta$ & $T_{\text {Miami }}$ & NRMSE \\
\hline 52 & Brandt & 0.28 & 0.02 & 0.07 & 0.58 & 1.14 & 0.12 \\
\hline 63 & Brandt & 0.27 & 0.02 & 0.09 & 0.64 & 0.78 & 0.12 \\
\hline 56 & Brandt & 0.24 & 0.03 & 0.04 & 0.60 & 1.11 & 0.13 \\
\hline 47 & Brandt & 0.21 & 0.04 & 0.02 & 0.46 & 2.31 & 0.13 \\
\hline 45 & Brandt & 0.23 & 0.02 & 0.05 & 0.80 & 0.23 & 0.13 \\
\hline 55 & Brandt & 0.25 & 0.05 & 0.03 & 0.73 & 0.44 & 0.14 \\
\hline 44 & Brandt & 0.37 & 0.02 & 0.10 & 0.55 & 1.10 & 0.15 \\
\hline 58 & Brandt & 0.35 & 0.04 & 0.01 & 0.66 & 0.79 & 0.15 \\
\hline 49 & Brandt & 0.36 & 0.04 & 0.05 & 0.71 & 0.54 & 0.16 \\
\hline 54 & Brandt & 0.33 & 0.02 & 0.08 & 0.90 & 0.09 & 0.16 \\
\hline 46 & Brandt & 0.32 & 0.03 & 0.07 & 0.93 & 0.08 & 0.17 \\
\hline 51 & Brandt & 0.39 & 0.05 & 0.01 & 0.46 & 2.55 & 0.17 \\
\hline 59 & Brandt & 0.40 & 0.03 & 0.04 & 0.78 & 0.31 & 0.17 \\
\hline 48 & Brandt & 0.46 & 0.03 & 0.06 & 0.41 & 3.17 & 0.18 \\
\hline 50 & Brandt & 0.30 & 0.04 & 0.02 & 0.99 & 0.05 & 0.20 \\
\hline 57 & Brandt & 0.49 & 0.02 & 0.00 & 0.68 & 0.60 & 0.20 \\
\hline 62 & Brandt & 0.43 & 0.02 & 0.03 & 0.82 & 0.17 & 0.21 \\
\hline 53 & Brandt & 0.47 & 0.05 & 0.06 & 0.51 & 1.91 & 0.22 \\
\hline 3 & Slinn droplet $=0.5 \mathrm{~mm}$ & 0.23 & 0.02 & 0.05 & 0.80 & 0.22 & 0.23 \\
\hline 43 & Brandt & 0.50 & 0.04 & 0.01 & 0.66 & 0.79 & 0.23 \\
\hline 14 & Slinn droplet $=0.5 \mathrm{~mm}$ & 0.24 & 0.03 & 0.04 & 0.60 & 1.03 & 0.24 \\
\hline 5 & Slinn droplet $=0.5 \mathrm{~mm}$ & 0.21 & 0.04 & 0.02 & 0.46 & 2.15 & 0.24 \\
\hline 21 & Slinn droplet $=0.5 \mathrm{~mm}$ & 0.27 & 0.02 & 0.09 & 0.64 & 0.73 & 0.24 \\
\hline 10 & Slinn droplet $=0.5 \mathrm{~mm}$ & 0.28 & 0.02 & 0.07 & 0.58 & 1.07 & 0.24 \\
\hline 61 & Brandt & 0.44 & 0.04 & 0.09 & 0.87 & 0.16 & 0.25 \\
\hline 60 & Brandt & 0.41 & 0.03 & 0.08 & 0.96 & 0.07 & 0.26 \\
\hline 13 & Slinn droplet $=0.5 \mathrm{~mm}$ & 0.25 & 0.05 & 0.03 & 0.73 & 0.41 & 0.27 \\
\hline 12 & Slinn droplet $=0.5 \mathrm{~mm}$ & 0.33 & 0.02 & 0.08 & 0.90 & 0.09 & 0.30 \\
\hline 2 & Slinn droplet $=0.5 \mathrm{~mm}$ & 0.37 & 0.02 & 0.10 & 0.55 & 1.04 & 0.30 \\
\hline 16 & Slinn droplet $=0.5 \mathrm{~mm}$ & 0.35 & 0.04 & 0.01 & 0.66 & 0.74 & 0.30 \\
\hline 4 & Slinn droplet $=0.5 \mathrm{~mm}$ & 0.32 & 0.03 & 0.07 & 0.93 & 0.08 & 0.31 \\
\hline 7 & Slinn droplet $=0.5 \mathrm{~mm}$ & 0.36 & 0.04 & 0.05 & 0.71 & 0.50 & 0.31 \\
\hline 17 & Slinn droplet $=0.5 \mathrm{~mm}$ & 0.40 & 0.03 & 0.04 & 0.78 & 0.29 & 0.32 \\
\hline 9 & Slinn droplet $=0.5 \mathrm{~mm}$ & 0.39 & 0.05 & 0.01 & 0.46 & 2.37 & 0.33 \\
\hline 15 & Slinn droplet $=0.5 \mathrm{~mm}$ & 0.49 & 0.02 & 0.00 & 0.68 & 0.56 & 0.34 \\
\hline 20 & Slinn droplet $=0.5 \mathrm{~mm}$ & 0.43 & 0.02 & 0.03 & 0.82 & 0.16 & 0.35 \\
\hline 6 & Slinn droplet $=0.5 \mathrm{~mm}$ & 0.46 & 0.03 & 0.06 & 0.41 & 2.95 & 0.35 \\
\hline 8 & Slinn droplet $=0.5 \mathrm{~mm}$ & 0.30 & 0.04 & 0.02 & 0.99 & 0.05 & 0.37 \\
\hline 11 & Slinn droplet $=0.5 \mathrm{~mm}$ & 0.47 & 0.05 & 0.06 & 0.51 & 1.78 & 0.39 \\
\hline 1 & Slinn droplet $=0.5 \mathrm{~mm}$ & 0.50 & 0.04 & 0.01 & 0.66 & 0.74 & 0.39 \\
\hline 19 & Slinn droplet $=0.5 \mathrm{~mm}$ & 0.44 & 0.04 & 0.09 & 0.87 & 0.15 & 0.43 \\
\hline
\end{tabular}


Table 4. Continued.

\begin{tabular}{llllllll}
\hline \multirow{2}{*}{ Expt ID } & Removal Scheme & mfpar $_{\text {lim }}$ & $\mathrm{sm}_{\text {lim }}$ & $\mathrm{sd}_{\text {lim }}$ & $\eta$ & $T_{\text {Miami }}$ & NRMSE \\
\hline 18 & Slinn droplet $=0.5 \mathrm{~mm}$ & 0.41 & 0.03 & 0.08 & 0.96 & 0.06 & 0.43 \\
24 & Slinn droplet $=0.1 \mathrm{~mm}$ & 0.23 & 0.02 & 0.05 & 0.80 & 0.18 & 0.46 \\
35 & Slinn droplet $=0.1 \mathrm{~mm}$ & 0.24 & 0.03 & 0.04 & 0.60 & 0.87 & 0.48 \\
42 & Slinn droplet $=0.1 \mathrm{~mm}$ & 0.27 & 0.02 & 0.09 & 0.64 & 0.61 & 0.48 \\
31 & Slinn droplet $=0.1 \mathrm{~mm}$ & 0.28 & 0.02 & 0.07 & 0.58 & 0.89 & 0.48 \\
26 & Slinn droplet $=0.1 \mathrm{~mm}$ & 0.21 & 0.04 & 0.02 & 0.46 & 1.81 & 0.48 \\
34 & Slinn droplet $=0.1 \mathrm{~mm}$ & 0.25 & 0.05 & 0.03 & 0.73 & 0.35 & 0.54 \\
23 & Slinn droplet $=0.1 \mathrm{~mm}$ & 0.37 & 0.02 & 0.10 & 0.55 & 0.88 & 0.55 \\
37 & Slinn droplet $=0.1 \mathrm{~mm}$ & 0.35 & 0.04 & 0.01 & 0.66 & 0.63 & 0.57 \\
33 & Slinn droplet $=0.1 \mathrm{~mm}$ & 0.33 & 0.02 & 0.08 & 0.90 & 0.07 & 0.57 \\
28 & Slinn droplet $=0.1 \mathrm{~mm}$ & 0.36 & 0.04 & 0.05 & 0.71 & 0.43 & 0.59 \\
25 & Slinn droplet $=0.1 \mathrm{~mm}$ & 0.32 & 0.03 & 0.07 & 0.93 & 0.07 & 0.60 \\
38 & Slinn droplet $=0.1 \mathrm{~mm}$ & 0.40 & 0.03 & 0.04 & 0.78 & 0.25 & 0.60 \\
30 & Slinn droplet $=0.1 \mathrm{~mm}$ & 0.39 & 0.05 & 0.01 & 0.46 & 2.04 & 0.61 \\
36 & Slinn droplet $=0.1 \mathrm{~mm}$ & 0.49 & 0.02 & 0.00 & 0.68 & 0.48 & 0.62 \\
41 & Slinn droplet $=0.1 \mathrm{~mm}$ & 0.43 & 0.02 & 0.03 & 0.82 & 0.14 & 0.64 \\
27 & Slinn droplet $=0.1 \mathrm{~mm}$ & 0.46 & 0.03 & 0.06 & 0.41 & 2.53 & 0.64 \\
29 & Slinn droplet $=0.1 \mathrm{~mm}$ & 0.30 & 0.04 & 0.02 & 0.99 & 0.04 & 0.65 \\
32 & Slinn droplet $=0.1 \mathrm{~mm}$ & 0.47 & 0.05 & 0.06 & 0.51 & 1.55 & 0.69 \\
22 & Slinn droplet $=0.1 \mathrm{~mm}$ & 0.50 & 0.04 & 0.01 & 0.66 & 0.65 & 0.70 \\
39 & Slinn droplet $=0.1 \mathrm{~mm}$ & 0.41 & 0.03 & 0.08 & 0.96 & 0.06 & 0.77 \\
40 & Slinn droplet $=0.1 \mathrm{~mm}$ & 0.44 & 0.04 & 0.09 & 0.87 & 0.13 & 0.77 \\
\hline & & & & & & & \\
\hline
\end{tabular}

Table 5. Tuning experiments ranked according to NRMSE with surface concentrations.

\begin{tabular}{llllllll}
\hline Expt ID & Removal Scheme & mfpar $_{\text {lim }}$ & $\mathrm{sm}_{\text {lim }}$ & $\mathrm{sd}_{\text {lim }}$ & $\eta$ & $T_{\text {Miami }}$ & NRMSE \\
\hline 29 & Slinn droplet $=0.1 \mathrm{~mm}$ & 0.30 & 0.04 & 0.02 & 0.99 & 8.40 & 0.28 \\
25 & Slinn droplet $=0.1 \mathrm{~mm}$ & 0.32 & 0.03 & 0.07 & 0.93 & 5.18 & 0.28 \\
39 & Slinn droplet $=0.1 \mathrm{~mm}$ & 0.41 & 0.03 & 0.08 & 0.96 & 5.99 & 0.28 \\
40 & Slinn droplet $=0.1 \mathrm{~mm}$ & 0.44 & 0.04 & 0.09 & 0.87 & 2.55 & 0.29 \\
34 & Slinn droplet $=0.1 \mathrm{~mm}$ & 0.25 & 0.05 & 0.03 & 0.73 & 0.97 & 0.30 \\
28 & Slinn droplet $=0.1 \mathrm{~mm}$ & 0.36 & 0.04 & 0.05 & 0.71 & 0.83 & 0.30 \\
38 & Slinn droplet $=0.1 \mathrm{~mm}$ & 0.40 & 0.03 & 0.04 & 0.78 & 1.41 & 0.30 \\
37 & Slinn droplet $=0.1 \mathrm{~mm}$ & 0.35 & 0.04 & 0.01 & 0.66 & 0.58 & 0.31 \\
35 & Slinn droplet $=0.1 \mathrm{~mm}$ & 0.24 & 0.03 & 0.04 & 0.60 & 0.43 & 0.31 \\
33 & Slinn droplet $=0.1 \mathrm{~mm}$ & 0.33 & 0.02 & 0.08 & 0.90 & 4.77 & 0.31 \\
26 & Slinn droplet $=0.1 \mathrm{~mm}$ & 0.21 & 0.04 & 0.02 & 0.46 & 0.20 & 0.32 \\
22 & Slinn droplet $=0.1 \mathrm{~mm}$ & 0.50 & 0.04 & 0.01 & 0.66 & 0.56 & 0.32 \\
24 & Slinn droplet $=0.1 \mathrm{~mm}$ & 0.23 & 0.02 & 0.05 & 0.80 & 1.97 & 0.32 \\
32 & Slinn droplet $=0.1 \mathrm{~mm}$ & 0.47 & 0.05 & 0.06 & 0.51 & 0.24 & 0.32 \\
42 & Slinn droplet $=0.1 \mathrm{~mm}$ & 0.27 & 0.02 & 0.09 & 0.64 & 0.62 & 0.32 \\
30 & Slinn droplet $=0.1 \mathrm{~mm}$ & 0.39 & 0.05 & 0.01 & 0.46 & 0.18 & 0.32 \\
27 & Slinn droplet $=0.1 \mathrm{~mm}$ & 0.46 & 0.03 & 0.06 & 0.41 & 0.15 & 0.32 \\
31 & Slinn droplet $=0.1 \mathrm{~mm}$ & 0.28 & 0.02 & 0.07 & 0.58 & 0.44 & 0.33 \\
41 & Slinn droplet $=0.1 \mathrm{~mm}$ & 0.43 & 0.02 & 0.03 & 0.82 & 2.63 & 0.34 \\
36 & Slinn droplet $=0.1 \mathrm{~mm}$ & 0.49 & 0.02 & 0.00 & 0.68 & 0.80 & 0.36 \\
23 & Slinn droplet $=0.1 \mathrm{~mm}$ & 0.37 & 0.02 & 0.10 & 0.55 & 0.45 & 0.37 \\
44 & Brandt & 0.37 & 0.02 & 0.10 & 0.55 & 0.77 & 0.38 \\
54 & Brandt & 0.33 & 0.02 & 0.08 & 0.90 & 7.61 & 0.39 \\
62 & Brandt & 0.43 & 0.02 & 0.03 & 0.82 & 4.22 & 0.40 \\
52 & Brandt & 0.28 & 0.02 & 0.07 & 0.58 & 0.69 & 0.41 \\
60 & Brandt & 0.03 & 0.08 & 0.96 & 9.24 & 0.42 \\
\hline & & 0.41 & & & & &
\end{tabular}



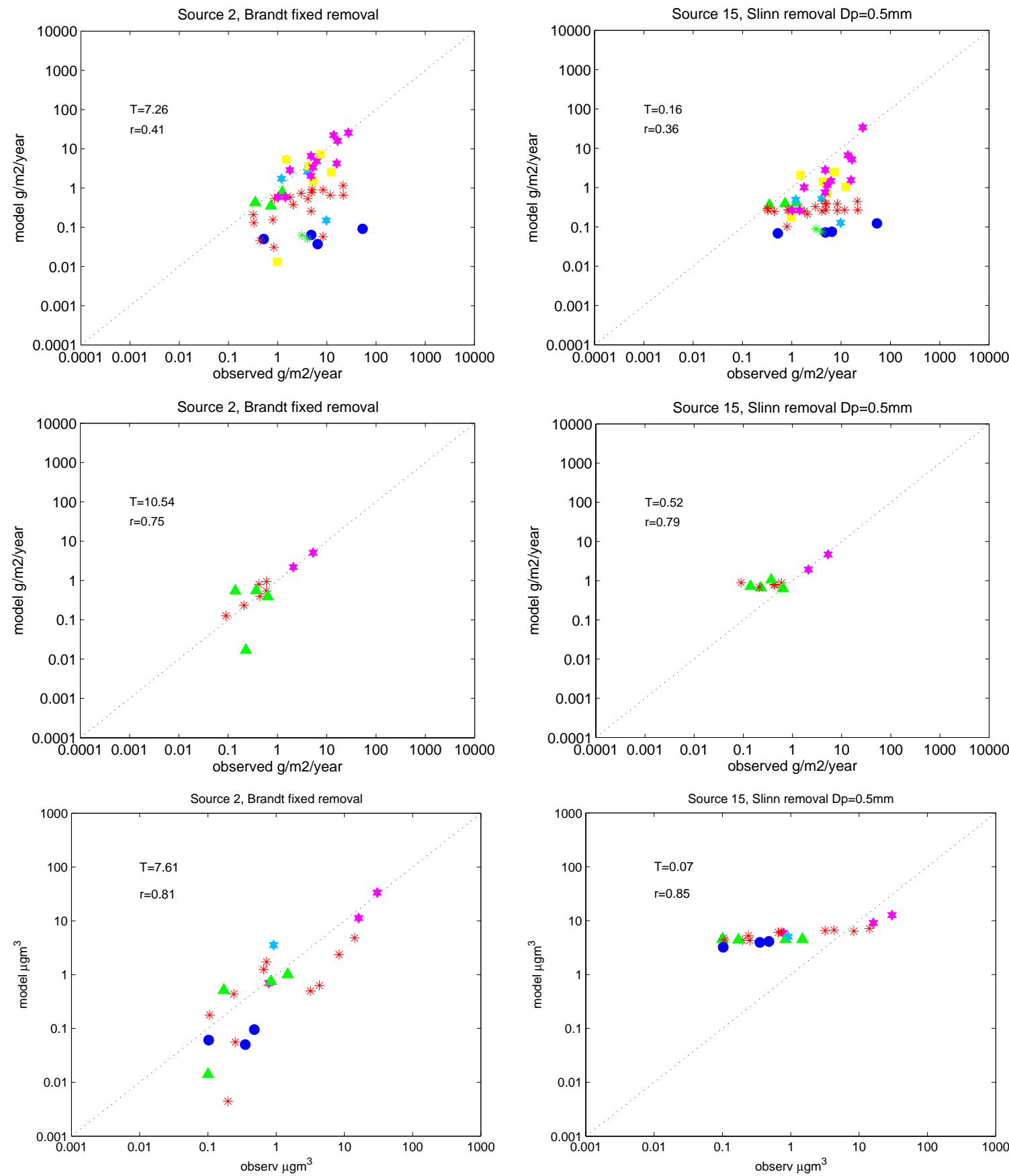

Fig. 10. A comparison between model output and DIRTMAP deposition (top), Ginoux deposition (middle) and surface concentration data (bottom). Data from the best the worst experiment (54) is shown on the left hand side and the worst experiment (11) on the right hand side. The location of measurement sites are denoted by colour; South Pacific (green), Antarctica/Southern Ocean (navy blue), North Pacific (red), North Atlantic (magenta), South Atlantic (pale blue), Arabian Sea (yellow).

The relationship between soil moisture and dust emissions in the model is currently very simple. Emissions are cut off if a threshold soil moisture is exceeded. This could be improved by using the scheme of Fécan et al. (1999) to calculate the increase in the threshold friction velocity due to the presence of soil moisture.
Another limitation of the model is that 6 hourly wind speeds are used to drive dust emissions. Although, this is the shortest time step the ERA-40 reanalysis data is available, it means that sporadic peaks in emissions which occur over short time periods are not captured. To improve this, a parameterisation for sub-grid scale gustiness could be used. A possible way to do this is to apply a probability distribution 
Table 5. Continued.

\begin{tabular}{|c|c|c|c|c|c|c|c|}
\hline Expt ID & Removal Scheme & mfpar $_{\text {lim }}$ & $\mathrm{sm}_{\lim }$ & $\mathrm{sd}_{\mathrm{lim}}$ & $\eta$ & $T_{\text {Miami }}$ & NRMSE \\
\hline 46 & Brandt & 0.32 & 0.03 & 0.07 & 0.93 & 7.83 & 0.42 \\
\hline 57 & Brandt & 0.49 & 0.02 & 0.00 & 0.68 & 1.27 & 0.42 \\
\hline 63 & Brandt & 0.27 & 0.02 & 0.09 & 0.64 & 0.95 & 0.42 \\
\hline 45 & Brandt & 0.23 & 0.02 & 0.05 & 0.80 & 3.01 & 0.42 \\
\hline 48 & Brandt & 0.46 & 0.03 & 0.06 & 0.41 & 0.23 & 0.43 \\
\hline 50 & Brandt & 0.30 & 0.04 & 0.02 & 0.99 & 12.54 & 0.43 \\
\hline 59 & Brandt & 0.40 & 0.03 & 0.04 & 0.78 & 2.08 & 0.44 \\
\hline 61 & Brandt & 0.44 & 0.04 & 0.09 & 0.87 & 3.84 & 0.44 \\
\hline 49 & Brandt & 0.36 & 0.04 & 0.05 & 0.71 & 1.22 & 0.44 \\
\hline 53 & Brandt & 0.47 & 0.05 & 0.06 & 0.51 & 0.36 & 0.44 \\
\hline 56 & Brandt & 0.24 & 0.03 & 0.04 & 0.60 & 0.63 & 0.44 \\
\hline 43 & Brandt & 0.50 & 0.04 & 0.01 & 0.66 & 0.84 & 0.45 \\
\hline 51 & Brandt & 0.39 & 0.05 & 0.01 & 0.46 & 0.27 & 0.45 \\
\hline 58 & Brandt & 0.35 & 0.04 & 0.01 & 0.66 & 0.84 & 0.45 \\
\hline 47 & Brandt & 0.21 & 0.04 & 0.02 & 0.46 & 0.30 & 0.46 \\
\hline 55 & Brandt & 0.25 & 0.05 & 0.03 & 0.73 & 1.38 & 0.46 \\
\hline 13 & Slinn droplet $=0.5 \mathrm{~mm}$ & 0.25 & 0.05 & 0.03 & 0.73 & 0.34 & 0.72 \\
\hline 3 & Slinn droplet $=0.5 \mathrm{~mm}$ & 0.23 & 0.02 & 0.05 & 0.80 & 0.71 & 0.72 \\
\hline 5 & Slinn droplet $=0.5 \mathrm{~mm}$ & 0.21 & 0.04 & 0.02 & 0.46 & 0.07 & 0.72 \\
\hline 14 & Slinn droplet $=0.5 \mathrm{~mm}$ & 0.24 & 0.03 & 0.04 & 0.60 & 0.15 & 0.73 \\
\hline 21 & Slinn droplet $=0.5 \mathrm{~mm}$ & 0.27 & 0.02 & 0.09 & 0.64 & 0.21 & 0.74 \\
\hline 4 & Slinn droplet $=0.5 \mathrm{~mm}$ & 0.32 & 0.03 & 0.07 & 0.93 & 1.68 & 0.74 \\
\hline 8 & Slinn droplet $=0.5 \mathrm{~mm}$ & 0.30 & 0.04 & 0.02 & 0.99 & 2.69 & 0.74 \\
\hline 10 & Slinn droplet $=0.5 \mathrm{~mm}$ & 0.28 & 0.02 & 0.07 & 0.58 & 0.14 & 0.74 \\
\hline 16 & Slinn droplet $=0.5 \mathrm{~mm}$ & 0.35 & 0.04 & 0.01 & 0.66 & 0.19 & 0.74 \\
\hline 7 & Slinn droplet $=0.5 \mathrm{~mm}$ & 0.36 & 0.04 & 0.05 & 0.71 & 0.27 & 0.75 \\
\hline 17 & Slinn droplet $=0.5 \mathrm{~mm}$ & 0.40 & 0.03 & 0.04 & 0.78 & 0.45 & 0.75 \\
\hline 12 & Slinn droplet $=0.5 \mathrm{~mm}$ & 0.33 & 0.02 & 0.08 & 0.90 & 1.54 & 0.75 \\
\hline 9 & Slinn droplet $=0.5 \mathrm{~mm}$ & 0.39 & 0.05 & 0.01 & 0.46 & 0.06 & 0.76 \\
\hline 19 & Slinn droplet $=0.5 \mathrm{~mm}$ & 0.44 & 0.04 & 0.09 & 0.87 & 0.75 & 0.76 \\
\hline 18 & Slinn droplet $=0.5 \mathrm{~mm}$ & 0.41 & 0.03 & 0.08 & 0.96 & 1.75 & 0.76 \\
\hline 20 & Slinn droplet $=0.5 \mathrm{~mm}$ & 0.43 & 0.02 & 0.03 & 0.82 & 0.81 & 0.77 \\
\hline 1 & Slinn droplet $=0.5 \mathrm{~mm}$ & 0.50 & 0.04 & 0.01 & 0.66 & 0.16 & 0.77 \\
\hline 2 & Slinn droplet $=0.5 \mathrm{~mm}$ & 0.37 & 0.02 & 0.10 & 0.55 & 0.14 & 0.77 \\
\hline 11 & Slinn droplet $=0.5 \mathrm{~mm}$ & 0.47 & 0.05 & 0.06 & 0.51 & 0.07 & 0.77 \\
\hline 6 & Slinn droplet $=0.5 \mathrm{~mm}$ & 0.46 & 0.03 & 0.06 & 0.41 & 0.04 & 0.77 \\
\hline 15 & Slinn droplet $=0.5 \mathrm{~mm}$ & 0.49 & 0.02 & 0.00 & 0.68 & 0.24 & 0.78 \\
\hline
\end{tabular}

function to the wind speed data (Grini et al., 2005; Cakmur et al., 2006). In the dry deposition scheme dust particles are prohibited from falling more than one model level per time step. Allowing particles to fall through multiple levels would improve the way in which larger particles are transported. Furthermore, including in-cloud scavenging as a mechanism of removal is not currently included in the model.

The LPJ-dust model has several potential applications. The model can be used to test whether vegetation changes can explain the observed variability in the dust loading on decadal time scales. This may help us distinguish between natural variability in dust cycle from anthropogenic effects such as land degradation. The model can also be used to study the dust cycle in the past. Ice core records show there has been a 2-25 fold increase in dust deposition rates during glacial periods compared to inter-glacial periods (Lambert et al., 2008). Previous studies have used focused on simulating the dust cycle at the LGM using the BIOME4 model in order to understand the reasons for the high dust loadings (Mahowald et al., 1999; Mahowald, 2006; Werner et al., 2002). The LPJ-dust model could be used to study the impact of dynamic vegetation on the dust loading through a deglaciation period. Likewise, the model can be used to investigate how dust sources will respond in the future with elevated atmospheric $\mathrm{CO}_{2}$ levels. Modelling studies using BIOME4 have shown that if vegetation cover is allowed to 
respond to elevated $\mathrm{CO}_{2}$ then dust emissions will decrease in the future (Mahowald and Luo, 2003; Mahowald, 2006, 2007). Using the LPJ-dust model would make it possible to predict the year to year variability in dust emissions in the future which is not possible using equilibrium vegetation models.

Acknowledgements. This study was funded by the Marie Curie GREENCYCLES Research and Training Network, FP6 (MRTNCT-2004-512464).

Edited by: A. Stenke

\section{References}

Brandt, J., Christensen, J. H., and Frohn, L. M.: Modelling transport and deposition of caesium and iodine from the Chernobyl accident using the DREAM model, Atmos. Chem. Phys., 2, 397-417, doi:10.5194/acp-2-397-2002, 2002.

Cakmur, R. V., Miller, R. L., Perlwitz, J., Geogdzhayev, I. V., Ginoux, P., Koch, D., Kohfeld, K. E., Tegen, I., and Zender, C. S.: Constraining the magnitude of the global dust cycle by minimizing the difference between a model and observations, J. Geophys. Res.-Atmos., 111, D06207, doi:10.1029/2005JD005791, 2006.

Chipperfield, M. P.: New version of the TOMCAT/SLIMCAT offline chemical transport model: Intercomparison of stratospheric tracer experiments, Q. J. Roy. Meteor. Soc., 132, 1179-1203, 2006.

Claquin, T., Schulz, M., and Balkanski, Y. J.: Modeling the mineralogy of atmospheric dust sources, J. Geophys. Res.-Atmos., 104(D18), 22243-22256, doi:10.1029/1999JD900416, 1999.

Coale, K. H., Johnson, K. S., Chavez, F. P., Buesseler, K. O., Barber, R. T., Brzezinski, M. A., Cochlan, W. P., Millero, F. J., Falkowski, P. G., Bauer, J. E., Wanninkhof, R. H., Kudela, R. M., Altabet, M. A., Hales, B. E., Takahashi, T., Landry, M. R., Bidigare, R. R., Wang, X. J., Chase, Z., Strutton, P. G., Friederich, G. E., Gorbunov, M. Y., Lance, V. P., Hilting, A. K., Hiscock, M. R., Demarest, M., Hiscock, W. T., Sullivan, K. F., Tanner, S. J., Gordon, R. M., Hunter, C. N., Elrod, V. A., Fitzwater, S. E., Jones, J. L., Tozzi, S., Koblizek, M., Roberts, A. E., Herndon, J., Brewster, J., Ladizinsky, N., Smith, G., Cooper, D., Timothy, D., Brown, S. L., Selph, K. E., Sheridan, C. C., Twining, B. S., and Johnson, Z. I.: Southern ocean iron enrichment experiment: Carbon cycling in high- and low-Si waters, Science, 304, 408-414, doi:10.1126/science.1089778, 2004.

Cramer, W., Kicklighter, D., and Bondeau, A.: Comparing global models of terrestrial net primary productivity (NPP): overview and key results, Glob. Change Biol., 5(S1), 1-16, doi:10.1046/j.1365-2486.1999.00007.x, 1999.

Edwards, N. and Marsh, R.: Uncertainties due to transportparameter sensitivity in an efficient 3-D ocean-climate model, Clim. Dynam., 24(4), 415-433, doi:10.1007/s00382-004-05088, 2005.

Evan, A. T., Heidinger, A. K., and Knippertz, P.: Analysis of winter dust activity off the coast of West Africa using a new 24year over-water advanced very high resolution radiometer satellite dust climatology, J. Geophys. Res.-Atmos., 111, D12210, doi:10.1029/2005JD006336, 2006.
Fécan, F., Marticorena, B., and Bergametti, G.: Parametrization of the increase of the aeolian erosion threshold wind friction velocity due to soil moisture for arid and semi-arid areas, Ann. Geophys., 17, 149-157, doi:10.1007/s00585-999-0149-7, 1999.

Forster, P., Ramaswamy, V., Artaxo, P., Berntsen, T., Betts, R., Fahey, D., Haywood, J., Lean, J., Lowe, D., Myhre, G., Nganga, J., Prinn, R., Raga, G., Schulz, M., and Dorland, R. V.: Changes in Atmospheric Constituents and in Radiative Forcing, in: Climate Change 2007: The Physical Science Basis, Contribution of Working Group I to the Fourth Assessment Report of the Intergovernmental Panel on Climate Change, Cambridge University Press, Cambridge and New York, NY, USA, 2007.

Ge, X. Z. and Lei, X. E.: Application and numerical experiments with a highly-accurate advection scheme in a regional transfer model, Meteorol. Atmos. Phys., 66(3-4), 131-142, doi:10.1007/BF01026629, 1998.

Gerten, D., Schaphoff, S., Haberlandt, U., Lucht, W., and Sitch, S.: Terrestrial vegetation and water balance - hydrological evaluation of a dynamic global vegetation model, J. Hydrol., 286(1-4), 249-270, doi:10.1016/j.jhydrol.2003.09.029, 2004.

Ginoux, P., Chin, M., Tegen, I., Prospero, J. M., Holben, B., Dubovik, O., and Lin, S. J.: Sources and distributions of dust aerosols simulated with the GOCART model, J. Geophys. Res.Atmos., 106, 20255-20273, doi:10.1029/2000JD000053, 2001.

Ginoux, P., Prospero, J. M., Torres, O., and Chin, M.: Long-term simulation of global dust distribution with the GOCART model: correlation with North Atlantic Oscillation, Environ. Modell. Softw., 19(2), 113-128, doi:10.1016/S1364-8152(03)00114-2, 2004.

Grini, A., Myhre, G., Zender, C. S., and Isaksen, I. S. A.: Model simulations of dust sources and transport in the global atmosphere: Effects of soil erodibility and wind speed variability, J. Geophys. Res., 110, D02205, doi:10.1029/2004JD005037, 2005.

Haxeltine, A. and Prentice, I. C.: A general model for the light-use efficiency of primary production, Funct. Ecol., 10(5), 551-561, 1996.

Haxeltine, A., Prentice, I. C., and Creswell, D. I.: A coupled carbon and water flux model to predict vegetation structure, J. Veg. Sci., 7(5), 651-666, doi:10.2307/3236377, 1996.

Herrmann, S. M., Anyamba, A., and Tucker, C. J.: Recent trends in vegetation dynamics in the African Sahel and their relationship to climate, Global Environ. Chang., 15, 394-404, 2005.

Huang, S., Titus, S., and Wiens, D.: Comparison of nonlinear height-diameter functions for major Alberta tree species, Can. J. Forest Res., 22, 1297-1304, 1992.

Iversen, J. D. and White, B. R.: Saltation Threshold on Earth, Mars and Venus, Sedimentology, 29, 111-119, 1982.

Jickells, T. D., An, Z. S., Andersen, K. K., Baker, A. R., Bergametti, G., Brooks, N., Cao, J. J., Boyd, P. W., Duce, R. A., Hunter, K. A., Kawahata, H., Kubilay, N., laRoche, J., Liss, P. S., Mahowald, N., Prospero, J. M., Ridgwell, A. J., Tegen, I., and Torres, R.: Global Iron Connections Between Desert Dust, Ocean Biogeochemistry, and Climate, Science, 308, 6771, doi:10.1126/science.1105959, 2005.

Joos, F., Gerber, S., Prentice, I. C., Otto-Bliesner, B. L., and Valdes, P. J.: Transient simulations of Holocene atmospheric carbon dioxide and terrestrial carbon since the Last Glacial Maximum, Global Biogeochem. Cy., 18, GB2002, doi:10.1029/2003GB002156, 2004. 
Jung, E. and Shao, Y.: An intercomparison of four wet deposition schemes used in dust transport modeling, Global Planet. Change, 52, 248-260, doi:10.1016/j.gloplacha.2006.02.008, 2006.

Kaufman, Y. J., Koren, I., Remer, L. A., Tanre, D., Ginoux, P., and Fan, S.: Dust transport and deposition observed from the Terra-Moderate Resolution Imaging Spectroradiometer (MODIS) spacecraft over the Atlantic ocean, J. Geophys. Res.Atmos., 110, D10S12, doi:10.1029/2003JD004436, 2005.

Kimura, R., Bai, L., and Wang, J. M.: Relationships among dust outbreaks, vegetation cover, and surface soil water content on the Loess Plateau of China, 1999-2000, Catena, 77, 292-296, iSI Document Delivery No.: 433XE, 2009.

Kohfeld, K. E. and Harrison, S. P.: DIRTMAP: The geological record of dust, Earth-Sci. Rev., 54(1-3), 81-114, doi:10.1016/S0012-8252(01)00042-3, 2001.

Lambert, F., Delmonte, B., Petit, J. R., Bigler, M., Kaufmann, P. R., Hutterli, M. A., Stocker, T. F., Ruth, U., Steffensen, J. P., and Maggi, V.: Dust-climate couplings over the past 800,000 years from the EPICA Dome C ice core, Nature, 452, 616-619, doi:10.1038/nature06763, 2008.

Lee, E.-H. and Sohn, B.-J.: Examining the impact of wind and surface vegetation on the Asian dust occurrence over three classified source regions, J. Geophys. Res., 114, D06205, doi:10.1029/2008JD010687, 2009.

Lindroth, A.: Aerodynamic and Canopy Resistance of Short-Rotation Forest in Relation to Leaf-Area Index and Climate, Bound.-Lay. Meteorol., 66(3), 265-279, doi:10.1007/BF00705478, 1993.

Louis, J. F.: A parametric model of vertical eddy fluxes in the atmosphere, Bound-Lay. Meteorol., 17(2), 187-202, doi:10.1007/BF00117978, 1979.

Lunt, D. J.: The mineral dust cycle at the last glacial maximum and the present day, Ph.D. thesis, University of Reading, UK, 2001.

Lunt, D. J. and Valdes, P. J.: The modern dust cycle: Comparison of model results with observations and study of sensitivities, J. Geophys. Res.-Atmos., 107(D23), 4669, doi:10.1029/2002JD002316, 2002.

Mahowald, N., Kohfeld, K., Hansson, M., Balkanski, Y., Harrison, S. P., Prentice, I. C., Schulz, M., and Rodhe, H.: Dust sources and deposition during the last glacial maximum and current climate: A comparison of model results with paleodata from ice cores and marine sediments, J. Geophys. Res., 104, 1589515916, doi:10.1029/1999JD900084, 1999.

Mahowald, N. M.: Change in atmospheric mineral aerosols in response to climate: Last glacial period, preindustrial, modern, and doubled carbon dioxide climates, J. Geophys. Res., 111, D10202, 0148-0227, doi:10.1029/2005JD006653, 2006.

Mahowald, N. M.: Anthropocene changes in desert area: Sensitivity to climate model predictions, Geophys. Res. Lett., 34, L18817, 10.1029/2007GL030472, 2007.

Mahowald, N. M. and Luo, C.: A less dusty future?, Geophys. Res. Lett., 30, 1903, doi:10.1029/2003GL017880, 2003.

Mahowald, N. M., Zender, C. S., Luo, C., Savoie, D., Torres, O., and del Corral, J.: Understanding the 30-year Barbados desert dust record, J. Geophys. Res.-Atmos., 107(D21), 4561, doi:10.1029/2002JD002097, 2002.

Marticorena, B. and Bergametti, G.: Modeling the atmospheric dust cycle: 1. Design of a soil-derived dust emission scheme, J. Geophys. Res., 100(D8), 16415-16430, doi:10.1029/95JD00690,
1995.

Marticorena, B., Bergametti, G., Aumont, B., Callot, Y., Ndoume, C., and Legrand, M.: Modeling the atmospheric dust cycle: 2. Simulation of Saharan dust sources, J. Geophys. Res., 102(D4), 4387-4404, doi:10.1029/96JD02964, 1997.

Matthews, E.: New high-resulation data sets for clmate studies, J. Appl. Meteorl., 22, 474-487, 1983.

McGuire, A., Sitch, S., and Clein, J.: Carbon balance of the terrestrial biosphere in the twentieth century: analyses of $\mathrm{CO}_{2}$, climate and land use effects with four process-based ecosystem models, Global Biogeochem. Cy., 15(1), 183-206, doi:10.1029/2000GB001298, 2001.

McKay, M. D., Beckman, R. J., and Conover, W. J.: A Comparison of Three Methods for Selecting Values of Input Variables in the Analysis of Output from a Computer Code, Technometrics, 21, 239-245, 1979.

Menendez, I., Diaz-Hernandez, J. L., Mangas, J., Alonso, I., and Sanchez-Soto, P. J.: Airborne dust accumulation and soil development in the North-East sector of Gran Canaria (Canary Islands, Spain), J. Arid Environ., 71, 57-81, 2007.

Monsi, M. and Saeki, T.: Über den Lichtfaktor in den Pflanzengesellschaften und seine Bedeutung fr die Stoffproduktion, Jpn. J. Bot., 14, 22-52, 1953 (in German).

Myhre, G., Grini, A., Haywood, J. M., Stordal, F., Chatenet, B., Tanré, D., Sundet, J. K., and Isaksen, I. S. A.: Modeling the radiative impact of mineral dust during the Saharan Dust Experiment (SHADE) campaign, J. Geophys. Res., 108(D18), 8579, doi:10.1029/2002JD002566, 2003.

Nicholson, S. E., Davenport, M. L., and Malo, A. R.: A Comparison of the Vegetation Response to Rainfall in the Sahel and East-Africa, Using Normalized Difference Vegetation Index from NOAA AVHRR, Climatic Change, 17, 209-241, doi:10.1007/BF00138369, 1990.

Prather, M. J.: Numerical Advection by Conservation of 2ndOrder Moments, J. Geophys. Res.-Atmos., 91(D6), 6671-6681, doi:10.1029/JD091iD06p06671, 1986.

Russell, G. L. and Lerner, J. A.: A New Finite-Differencing Scheme for the Tracer Transport-Equation, J. Appl. Meteorol., 20, 14831498, 1981.

Schneider von Deimling, T., Held, H., Ganopolski, A., and Rahmstorf, S.: Climate sensitivity estimated from ensemble simulations of glacial climate, Clim. Dynam., 27, 149-163, 2006.

Seaquist, J. W., Hickler, T., Eklundh, L., Ardö, J., and Heumann, B. W.: Disentangling the effects of climate and people on Sahel vegetation dynamics, Biogeosciences, 6, 469-477, doi:10.5194/bg6-469-2009, 2009.

Seinfeld, S. N. and Pandis, S. N.: Atmospheric Chemistry and Physics, from Air Polution to Climate Change, 2nd edn., Wiley, New York, 1998.

Sitch, S., Smith, B., Prentice, I., Arneth, A., Bondeau, A., Cramer, W., Kaplan, J. O., Levis, S., Lucht, W., Sykes, M., and Thonicke, K.: Evaluation of ecosystem dynamics, plant geography and terrestrial carbon cycling in the LPJ dynamic global vegetation model, Glob. Change Biol., 9, 161-185, 2003.

Slinn, W.: Precipitation scavenging, in: Atmospheric Sciences and Power Production-1979, US Department of Energy Office, Washington, DC, 1983.

Tanaka, T. Y. and Chiba, M.: A numerical study of the contributions of dust source regions to the global dust budget, Global Planet. 
Change, 52, 88-104, 2006.

Tegen, I. and Fung, I.: Modeling of Mineral Dust in the Atmosphere - Sources, Transport, and Optical-Thickness, J. Geophys. Res.Atmos., 99(D11), 22897-22914, doi:10.1029/94JD01928, 1994.

Tegen, I., Harrison, S. P., Kohfeld, K., Prentice, I. C., Coe, M., and Heimann, M.: Impact of vegetation and preferential source areas on global dust aerosol: Results from a model study, J. Geophys. Res.-Atmos., 107(D21), 4576, doi:10.1029/2001JD000963, 2002.

Tiedtke, M.: A comprehensive mass flux scheme for cumulus parameterization in large-scale models, Mon. Weather Rev., 8, 1779-1800, 1989.

Tucker, C. J., Dregne, H. E., and Newcomb, W. W.: Expansion and Contraction of the Sahara Desert from 1980 to 1990, Science, 253, 299-301, doi:10.1126/science.253.5017.299, 1991.

Wagner, W., Scipal, K., Pathe, C., Gerten, D., Lucht, W., and Rudolf, B.: Evaluation of the agreement between the first global remotely sensed soil moisture data with model and precipitation data, J. Geophys. Res.-Atmos., 108(D19), 4611, doi:10.1029/2003JD003663, 2003.

Werner, M., Tegen, I., Harrison, S. P., Kohfeld, K. E., Prentice, I. C., Balkanski, Y., Rodhe, H., and Roelandt, C.: Seasonal and interannual variability of the mineral dust cycle under present and glacial climate conditions, J. Geophys. Res.-Atmos., 107(D24), 4744, doi:10.1029/2002JD002365, 2002.
Yue, X., Wang, H. J., Wang, Z. F., and Fan, K.: Simulation of dust aerosol radiative feedback using the Global Transport Model of Dust: 1. Dust cycle and validation, J. Geophys. Res.-Atmos., 114, D10202, doi:10.1029/2008JD010995, 2009.

Zeide, B.: Primary unit of the tree crown, Ecology, 74, 1598-1602, 1993.

Zender, C. S. and Kwon, E. Y.: Regional contrasts in dust emission responses to climate, J. Geophys. Res., 110, D13201, doi:10.1029/2004JD005501, 2005.

Zender, C. S., Bian, H., and Newman, D.: Mineral Dust Entrainment and Deposition (DEAD) model: Description and 1990s dust climatology, J. Geophys. Res., 108(D14), 4416, doi:10.1029/2002JD002775, 2003.

Zhao, C. S.: Relationship between climatic factors and dust storm frequency in Inner Mongolia of China, Geophys. Res. Lett., 31, L01103, doi:10.1029/2003GL018351, 2004.

Zobler, L.: A world soil file for global climate modeling, Tech. Rep. TM87802, NASA, 1986. 